\title{
The index of linear differential algebraic equations with properly stated leading terms
}

\author{
R. März
}

\section{Introduction}

A linear differential algebraic equation (DAE) with properly stated leading term is of the form

$$
A(t)(D(t) x(t))^{\prime}+B(t) x(t)=q(t)
$$

with in some sense well matched coefficients $A(t)$ and $D(t)$. The coefficients are supposed to be continuous in $t$ matrix functions $A(t) \in L\left(\mathbb{R}^{n}, \mathbb{R}^{m}\right), D(t) \in L\left(\mathbb{R}^{m}, \mathbb{R}^{n}\right)$, $B(t) \in L\left(\mathbb{R}^{m}\right)$. In contrast to a standard form DAE

$$
E(t) x^{\prime}(t)+F(t) x(t)=q(t),
$$

in (1.1), the leading term precisely figures out the actually involved derivatives.

In [BaMä], DAEs of the form (1.1) are introduced and studied in some detail. In particular, an index $\mu$ notion is characterized for $\mu \in\{1,2\}$. The aim of the present paper is to define an appropriate general index $\mu$ for (1.1) in terms of the coefficients $A, D$ and $B$. Clearly, in case of smooth coefficients, one could turn to the standard form DAE $A D x^{\prime}+\left(B-A D^{\prime}\right) x=q$ and apply well-known index notions. However, we set a high value on doing with coefficients supposed to be continuous only. Hence, index notions related to derivative array systems and reduction techniques (e.g. [Ca], [RaRh], [KuMe]) do not apply for smoothness reasons. Further, the tractability index (e.g. [Mä2]) is given only if $n=m$ and $D(t)$ represents a smooth projector matrix. Here we do not assume any of the coefficients to be projectors, but $A$ and $D$ may actually be of rectangular size.

It should be mentioned that linear and nonlinear DAEs with properly stated leading term arise e.g. in circuit simulation. Furthermore, as observed recently, numerical methods applied to a DAE with properly stated leading term often work better than those applied to a standard DAE (e.g. [Mä], [HiMäTi]).

Further, the adjoint equation to (1.1)

$$
D^{*}(t)\left(A^{*}(t) y(t)\right)^{\prime}-B^{*}(t) y(t)=r(t)
$$

has the same form while this is not the case for the standard form DAE (1.2) and its adjoint equation $\left(E^{*}(t) y(t)\right)^{\prime}-F^{*}(t) y(t)=r(t)$. This symmetry yields advantages in optimal control problems. Now, the DAEs to be controlled, their adjoints, and also the boundary value problems resulting from extremal conditions may be treated in a unified way. Furthermore, due to properly stated leading terms, the sensitivity analysis becomes easier and more transparent (cf. [Mä3]. [BaMä]). 
Hence, by various reasons we are led to study equations of the form (1.1) in more detail. It should be stressed once more that neither $A(t)$ nor $D(t)$ is assumed to be a projector while, in the framework of tractability index (e.g. [Mä1]), $D(t)$ has to be a smooth projector.

Constant coefficient standard DAEs

$$
E x^{\prime}(t)+F x(t)=q(t)
$$

with regular matrix pencils $\{E, F\}$ are best understood. The Kronecker index $\mu$ of (1.3) is defined to be equal to the index of the pencil, i.e., $\mu=i n d\{E, F\}$ (for matrix pencils see e.g. [Ga]). Sometimes this index is named after Weierstraß and Riesz, too. With projections $P_{E}, R_{E} \in L\left(\mathbb{R}^{m}\right)$, ker $P_{E}=k e r E, i m R_{E}=i m E$, the constant coefficient DAE (1.3) immediately may be rewritten with properly stated leading term as

$$
E\left(P_{E} x(t)\right)^{\prime}+F x(t)=q(t)
$$

but also as

$$
R_{E}(E x(t))^{\prime}+F x(t)=q(t) .
$$

Clearly, the index of (1.4) and (1.5), respectively, should be $\mu=\operatorname{ind}\{E, F\}$.

Now we transform the unknown function in (1.3) by $x(t)=H(t) \bar{x}(t)$. Provided that $H(t) \in L\left(\mathbb{R}^{m}\right)$ is nonsingular and depends continuously differentiably on $t$, we arrive at

$$
E H(t) \bar{x}^{\prime}(t)+\left(F H(t)+E H^{\prime}(t)\right) \bar{x}(t)=q(t) .
$$

There are different possibilities to reformulate (1.6) for getting a properly stated leading term. We have e.g. $E H(t) \bar{x}^{\prime}(t)=E\left(P_{E} H(t) \bar{x}(t)\right)^{\prime}-E H^{\prime}(t) \bar{x}(t)$, which leads to

$$
E\left(P_{E} H(t) \bar{x}(t)\right)^{\prime}+F H(t) \bar{x}(t)=q(t),
$$

and $E H(t) \bar{x}^{\prime}(t)=R_{E}\left(E H(t) \bar{x}^{\prime}(t)\right)^{\prime}-E H^{\prime}(t) \bar{x}(t)$, which leads to

$$
R_{E}\left(E H(t) \bar{x}^{\prime}(t)\right)^{\prime}+F H(t) \bar{x}(t)=q(t)
$$

i.e., we obtain the transformed versions of (1.4) and (1.5). On the other hand, using the relation $E H(t) \bar{x}^{\prime}(t)=E P_{E} H(t) \bar{x}^{\prime}(t)=E H(t) H(t)^{-1} P_{E} H(t) \bar{x}^{\prime}(t)=E H(t)\left(H(t)^{-1}\right.$ $\left.P_{E} H(t) \bar{x}(t)\right)^{\prime}-E H(t)\left(H(t)^{-1} P_{E} H(t)\right)^{\prime} \bar{x}(t)$ we may reformulate (1.6) as

$$
E H(t)\left(H(t)^{-1} P_{E} H(t) \bar{x}(t)\right)^{\prime}+\left(F H(t)+E(t) H^{\prime}(t) H(t)^{-1} P_{E} H(t)\right) \bar{x}(t)=q(t) .
$$

Observe that $H(t)^{-1} P_{E} H(t)$ is a smooth projector along $k e r(E H(t))$ such that (1.9) represents the form of DAEs considered in the context of the tractability index. No doubt, all those versions should have the same index $\mu=\operatorname{ind}\{E, F\}$.

An indirect index notion for (1.1) saying that (1.1) has index $\mu$ if this DAE results from a constant coefficient DAE which has index $\mu$ by transforming the unknown function and scaling the equation would be possible. In such a way, the so-called global index (or better, Kronecker index) of the time varying standard case (1.2) is given ([GePe]). However, we are interested in an index criterion that is formulated in terms of the coefficients $A, D, B$ and which can be applied in a more constructive way. 
In this paper, we will get along with continuous coefficients $A, D, B$ provided that certain characteristic subspaces are of class $C^{1}$, i.e., they are spanned by continuously differentiable functions.

If $A(t)$ and $D(t)$ remain nonsingular, equation (1.1) is actually an implicit regular ordinary differential equation (ODE), which may be rewritten as an explicit ODE for the product $D() x.($.$) , namely$

$$
(D(t) x(t))^{\prime}=-A(t)^{-1} B(t) D(t)^{-1} D(t) x(t)+A(t)^{-1} q(t) .
$$

Obviously, classical solutions of those equations belong to the class $C_{D}^{1}$ consisting of continuous functions $x($.$) having a continuously differentiable product D() x.($.$) . Below,$ we will apply this solution understanding in the case of singular coefficients $D(t)$, too.

A premultiplication of (1.10) by $H(t)^{-1}$ and taking $H(t)^{-1}(D(t) x(t))^{\prime}=$ $=\left(H(t)^{-1} D(t) x(t)\right)^{\prime}-H(t)^{-1 \prime} D(t) x(t)$ yields

$$
A(t) H(t)\left(H(t)^{-1} D(t) x(t)\right)^{\prime}+\left(B(t)+A(t) H^{\prime}(t) H(t)^{-1} D(t)\right) x(t)=q(t),
$$

which corresponds to the refactorization $A D=(A H)\left(H^{-1} D\right)$ of the leading term in (1.1). Recall that, for explicit ODEs, kinematic similarity transformations always consist of two steps, namely, transforming the unknown and premultiplying the vector field to obtain an explicit ODE again (e.g. [Gaj]). For $A=I, D=I$, the equations (1.1) and (1.11) simplify to $x^{\prime}+B x=q$ and $H\left(H^{-1} x\right)^{\prime}+\left(B+H^{\prime} H^{-1}\right) x=q$. Obviously, transforming $x=H \bar{x}$ and premultiplying by $H^{-1}$ yields $\bar{x}^{\prime}+H^{-1} B H \bar{x}+H^{-1} H^{\prime} \bar{x}=$ $H^{-1} q$. As we shall see below, the refactorization of the leading term realized in (1.11) is in general closely related to a respective transformation of the inherent in the DAE regular explicit ODE. In the consequence, the index notion we are looking for should be invariant under refactorizations, too.

In this paper, we give an index notion that includes the lower index cases considered in [BaMä] and [Schu] and, further, generalizes the so-called global index proposed in $[\mathrm{GePe}]$ as well as the tractability index.

In Section 2, for given coefficients $A, D$ and $B$, a special sequence of matrix functions is constructed so that an index notion can be realized in terms of these matrices.

In Section 3 we show what the inherent regular ODE looks like.

In Section 4 the index notion is shown to be invariant under linear regular transformations and under refactorizations of the leading term.

In Section 5 we attempt to relate the index notion given for (1.1) to different concepts introduced in the literature for smooth standard form DAEs (1.2). We end up with some concluding remarks. The Appendix contains technically expensive proofs.

\section{Matrix sequence and index}

Consider equations

$$
A(t)(D(t) x(t))^{\prime}+B(t) x(t)=q(t), \quad t \in I,
$$

with continuous matrix coefficients

$$
A(t) \in L\left(\mathbb{R}^{n}, \mathbb{R}^{m}\right), \quad D(t) \in L\left(\mathbb{R}^{m}, \mathbb{R}^{n}\right), \quad B(t) \in L\left(\mathbb{R}^{m}\right), \quad t \in I \subseteq \mathbb{R} .
$$


Definition 2.1 The leading term of (2.1) is stated properly if the coefficients $A(t)$ and $D(t)$ are well matched in the sense that

$$
\operatorname{ker} A(t) \oplus \operatorname{im} D(t)=\mathbb{R}^{n}, \quad t \in I,
$$

and there is a continuously differentiable with respect to $t$ projector $R(t) \in L\left(\mathbb{R}^{n}\right)$ such that $\operatorname{im} R(t)=\operatorname{im} D(t)$, $\operatorname{ker} R(t)=\operatorname{ker} A(t), t \in I$.

By definition, the matrices $A(t)$ and $D(t)$ in a properly stated leading term have a common constant rank.

Definition 2.2 A continuous function $x: I \rightarrow \mathbb{R}^{m}$ is said to be a solution of equation (2.1) if it has a continuously differentiable part $D x: I \rightarrow \mathbb{R}^{n}$ and equation (2.1) is satisfied pointwise.

Denote the corresponding function space by

$$
C_{D}^{1}\left(I, \mathbb{R}^{m}\right):=\left\{x \in C\left(I, \mathbb{R}^{m}\right): D x \in C^{1}\left(I, \mathbb{R}^{n}\right)\right\} .
$$

Next we form a sequence of matrix functions and possibly time-varying subspaces to be used frequently later on. All relations are ment pointwise for each $t \in I$, but we drop the argument $t$.

For given coefficients $A, D, B, A$ and $D$ well matched, we define

$$
\begin{aligned}
& G_{0}=A D, B_{-1}=B, P_{-1}=I, Q_{-1}=0, N_{0}=\operatorname{ker} G_{0} . \\
& Q_{0}, W_{0}: I \rightarrow L\left(\mathbb{R}^{m}\right) \text { denote projector functions such that } \\
& Q_{0}^{2}=Q_{0}, W_{0}^{2}=W_{0}, i m Q_{0}=N_{0}, \operatorname{ker} W_{0}=i m G_{0}, \\
& P_{0}=I-Q_{0} . \\
& D^{-}: I \rightarrow L\left(\mathbb{R}^{n}, \mathbb{R}^{m}\right) \text { denotes the reflexive generalized inverse of } D \text { such that } \\
& D^{-} D D^{-}=D^{-}, D D^{-} D=D, D D^{-}=R, D^{-} D=P_{0} .
\end{aligned}
$$

Further, for $i \geq 0$ :

$$
\begin{aligned}
B_{i} & =B_{i-1} P_{i-1}-G_{i} D^{-}\left(D P_{0} \cdots P_{i} D^{-}\right)^{\prime} D P_{-1} P_{0} \cdots P_{i-1}, \\
S_{i} & =\left\{z \in \mathbb{R}^{m}: B_{i} z \in i m G_{i}\right\}=\operatorname{ker} W_{i} B_{i}=\operatorname{ker} W_{i} B \\
G_{i+1} & =G_{i}+B_{i} Q_{i}, \\
N_{i+1} & =\operatorname{ker} G_{i+1}, \quad Q_{i+1}^{2}=Q_{i+1}, \quad i m Q_{i+1}=N_{i+1}, \quad P_{i+1}=I-Q_{i+1}, \\
W_{i+1}^{2} & =W_{i+1}, \operatorname{ker} W_{i+1}=i m G_{i+1} .
\end{aligned}
$$

For the moment, we assume the derivative used in the definition of $B_{i}$ to exist. We will resume this point later on. The idea to form just this sequence, in particular the special $B_{i}$, originates from the tractability index ([Mä1], [Mä2]).

Below, the sequence of matrix functions $G_{i}, i \geq 0$, will play a special role. Notice that the projectors $W_{j}$ are not involved at all in the definition of $G_{i}$. We shall make use of them in describing properties only. Observe that, due to $G_{i+1} P_{i}=G_{i}$, we may write $G_{i+1}$ as a product

$$
G_{i+1}=\left(G_{i}+B_{i-1} P_{i-1} Q_{i}\right)\left(I-P_{i} D^{-}\left(D P_{0} \cdots P_{i} D^{-}\right)^{\prime} D P_{0} \cdots P_{i-1} Q_{i}\right) .
$$


Since the second factor of this product is nonsingular, it holds that

$$
\operatorname{rank} G_{i+1}=\operatorname{rank}\left(G_{i}+B_{i-1} P_{i-1} Q_{i}\right) .
$$

Further, denoting by $G_{i}^{-}$the reflexive generalized inverse with $G_{i} G_{i}^{-}=I-W_{i}$ and $G_{i}^{-} G_{i}=P_{i}$, we may reformulate

$$
G_{i+1}=G_{i}+W_{i} B_{i} Q_{i}+\left(I-W_{i}\right) B_{i} Q_{i}=G_{i}+W_{i} B_{i-1} P_{i-1} Q_{i}+G_{i} G_{i}^{-} B_{i} Q_{i}
$$

and then factorize $G_{i+1}=\mathcal{G}_{i+1} \mathcal{F}_{i+1}$ with factors

$$
\mathcal{G}_{i+1}=G_{i}+W_{i} B_{i-1} P_{i-1} Q_{i}=G_{i}+W_{i} B Q_{i},
$$

and $\mathcal{F}_{i+1}=I+G_{i}^{-} B_{i-1} P_{i-1} Q_{i}-P_{i} D^{-}\left(D P_{0} \cdots P_{i} D^{-}\right)^{\prime} D P_{0} \cdots P_{i-1} Q_{i}$.

The factor $\mathcal{F}_{i+1}$ is always nonsingular, hence

$$
\operatorname{rank} G_{i+1}=\operatorname{rank} \mathcal{G}_{i+1}, \quad i m G_{i+1}=i m \mathcal{G}_{i+1}=i m G_{i} \oplus i m W_{i} B Q_{i} .
$$

By this we know the rank of the matrices $G_{i}$ to increase monotonously, i.e.,

$\operatorname{rank} G_{0} \leq \operatorname{rank} G_{1} \leq \ldots \leq \operatorname{rank} G_{i} \leq \ldots$, and more precisely, rank $G_{i+1}-\operatorname{rank} G_{i}=$ rank $W_{i} B Q_{i} \geq 0$.

Observe further that

$$
\begin{aligned}
& \text { ker } \mathcal{G}_{i+1}=N_{i} \cap S_{i}, \quad N_{i+1}=\mathcal{F}_{i+1}^{-1}\left(N_{i} \cap S_{i}\right), \\
& N_{i+1} \cap N_{i}=N_{i} \cap \operatorname{ker} B_{i} \subseteq N_{i+1} \cap \operatorname{ker} B_{i+1}=N_{i+2} \cap N_{i+1} .
\end{aligned}
$$

As a simple but important consequence of relation (2.6), a certain nontrivial intersection $N_{i_{*}+1} \cap N_{i_{*}}$ would yield the whole sequence $\left\{G_{k}\right\}_{k \geq 0}$ to consist of singular matrices only.

The following assertion concerns the constant coefficient case. It is an immediate consequence of [GrMä, Theorem 3].

Theorem 2.3 Let $A, D$ and $B$ be time-invariant, $A, D$ be well matched.

Then the matrix pencil $\{A D, B\}$ is regular with index $\mu$ if and only if $G_{0}, \ldots, G_{\mu-1}$ are singular, but $G_{\mu}$ is nonsingular.

By Theorem 2.3, the matrix sequence $G_{i}, i \geq 0$, provides an index criterion independently of the choice of the projectors $Q_{i}, i \geq 0$. For regular pencils it holds that $0 \leq \mu \leq m$. Further, because of (2.6), the nonsingularity of $G_{\mu}$ implies the relation

$$
N_{i+1} \cap N_{i}=0 \text { for all } i \geq 0 .
$$

If there is a nontrivial intersection $N_{i_{*}+1} \cap N_{i_{*}}$, the matrix pencil has to be a singular one. In particular, it is necessary for the regularity of the pencil $\{A D, B\}$ that $N_{0} \cap N_{1}=0$ is valid. But then, the projector $Q_{1}$ onto $N_{1}$ can be chosen so that $N_{0} \subset \operatorname{ker} Q_{1}$, thus $Q_{1} Q_{0}=0$. Constructing the sequence of matrices $G_{i}$, we may successively choose the projectors $Q_{i+1}$ so that $Q_{i+1} Q_{j}=0, j=0, \ldots, i$, holds true as long as $N_{i+1} \cap N_{j}=$ $0, j=0 \ldots, i$ (cf. [GrMä]).

Now we turn back to the time-varying case. Provided that the intersection

$$
N_{0} \cap N_{1}=N_{0} \cap \operatorname{ker} B_{0}=\operatorname{ker}(A D) \cap \operatorname{ker} B
$$


is trivial, we choose $Q_{1}$ so that $N_{0} \subset \operatorname{ker} Q_{1}$, i.e., $Q_{1} Q_{0}=0$. In the next step we suppose that $N_{1} \cap N_{2}=0$. Now, $z \in N_{2} \cap N_{0}$ implies $z=Q_{0} z, 0=G_{2} z=\left(G_{1}+\right.$ $\left.B_{1} Q_{1}\right) z=G_{1} z+B_{1} Q_{1} Q_{0} z=G_{1} z$, i.e., $z \in N_{0} \cap N_{1}=0$, thus $N_{2} \cap N_{0}=0$. Due to $N_{2} \cap N_{j}=0, j=0,1$, we choose $Q_{2}$ so that $N_{0} \oplus N_{1} \subset \operatorname{ker} Q_{2}$.

In general, let the projector up to index $i$ satisfy $Q_{j} Q_{k}=0, k=0, \ldots, j-1, j=1, \ldots, i$, and let $N_{i+1} \cap N_{i}=0$ be true. Then, for $k \in\{0, \ldots, i-1\}, z \in N_{i+1} \cap N_{k}$ implies $z=Q_{k} z, \quad 0=G_{i+1} z=G_{i} z+B_{i} Q_{i} Q_{k} z=G_{i} z=\ldots=G_{k+1} z$, hence $z \in N_{k} \cap N_{k+1}=0$, thus $N_{i+1} \cap N_{k}=0, k=0, \ldots, i-1$. This allows us to choose $Q_{i+1}$ in such a way that $N_{0} \oplus N_{1} \oplus \cdots \oplus N_{i} \subseteq \operatorname{ker} Q_{i+1}$,

$$
Q_{i+1} Q_{j}=0, \quad j=0, \ldots, i, i \geq 0,
$$

holds true. In the consequence, certain products of projectors also become projectors, e.g. $P_{0} P_{1} \cdots P_{i}, \quad P_{0} P_{1} \cdots P_{i-1} Q_{i}$ etc.

Recall once more that a certain nontrivial $N_{i_{*}+1} \cap N_{i_{*}}$ would yield the whole sequence $\left\{G_{k}\right\}_{k \geq 0}$ to consist of singular matrices only (cf. (2.6)).

Since $G_{0}(t)$ is continuous and has constant rank on $\mathcal{I}$, we may begin the sequence $(2.2)$ with a continuous in $t$ projector $Q_{0}(t)$. Then, a continuous $G_{1}(t)$ results. If it has also constant rank, or equivalently, if the intersection $N_{0}(t) \cap S_{0}(t)$ does not change its dimension (cf. (2.5)), then we may rely on a continuous subsequent projector $Q_{1}(t)$ and so on.

Due to condition (2.7), the decompositions $I=P_{0}+Q_{0}=P_{0} P_{1}+P_{0} Q_{1}+Q_{0}=P_{0} P_{1}$. $P_{i}+P_{0} \cdots P_{i-1} Q_{i}+\cdots+P_{0} Q_{1}+Q_{0}$ are realized by projectors acting on $\mathbb{R}^{m}$, i.e., under certain constant rank conditions the $\mathbb{R}^{m}$ is decomposed into continuous subspaces. Similarly, the terms in the decompositions

$$
\begin{aligned}
R=D D^{-} & =D P_{0} D^{-}=D P_{0} P_{1} D^{-}+D P_{0} Q_{1} D^{-} \\
& =D P_{0} P_{1} P_{2} D^{-}+D P_{0} P_{1} Q_{2} D^{-}+D P_{0} Q_{1} D^{-} \\
& =D P_{0} \cdots P_{i} D^{-}+D P_{0} \cdots P_{i-1} Q_{i} D^{-}+\cdots+D P_{0} Q_{1} D^{-}
\end{aligned}
$$

are projectors, too. Together with $I-R$, they decompose the $\mathbb{R}^{n}$ into continuous subspaces. Recall that $R$ is continuously differentiable by Definition 2.1. Below, additionally, we shall demand these continuous projectors $D P_{0} \cdots P_{i} D^{-}, i>0$, to be just continuously differentiable. In the consequence, $D P_{0} Q_{1} D^{-}=R-D P_{o} P_{1} D^{-}$also belongs to $C^{1}$ and so do all $D P_{0} \cdots P_{i-1} Q_{i} D^{-}, i \geq 1$. By this, the continuously differentiable subspace $i m D$ is decomposed into further continuously differentiable subspaces. Let us stress that this is the only additional smoothness condition we shall need.

The formal reason for assuming $D P_{0} \cdots P_{i} D^{-} \in C^{1}$ is the construction of $B_{i}$ in the matrix function sequence (2.2). However, there is a rather substantial background.

As discussed in [BaMä] and [Schu] for $1 \leq \mu \leq 3$, the subspace im $D P_{0} \cdots P_{\mu-1} D^{-}$is exactly the one in which the so-called inherent regular ODE of a DAE (2.1) with index $\mu$ has to be considered. This fact will be confirmed once more by Theorem 3.2 below, where the inherent regular ODE is figured out for the general index case. From this point of view, demanding that $D P_{0} \cdots P_{i} D^{-}$belongs to $C^{1}$ seems to be very natural.

If $G_{0}(t)$ remains nonsingular on $\mathcal{I}$, then equation (2.1) is actually an implicit ODE with solely regular points (e.g. [CoCa]), that is, a regular implicit ODE. Obviously, for each 
continuous inhomogeneity $q$, this equation (2.1) is solvable on $C_{D}^{1}\left(\mathcal{I}, \mathbb{R}^{m}\right)$. The corresponding homogeneous equation has an $m$-dimensional solution space. Multiplying (2.1) by $A(t)^{-1}$ leads to the inherent explicit regular ODE for the function $D(t) x(t)$.

The regular implicit ODE $\left((2.1)\right.$ with nonsingular $\left.G_{0}=A D\right)$ can be interpreted as a regular DAE with index $\mu=0$. Accordingly, with a regular index $\mu$ DAE, $\mu>0$, defined below, we associate the expectation of solvability on $C_{D}^{1}\left(\mathcal{I}, \mathbb{R}^{m}\right)$ at least for inhomogeneities $q \in C^{\mu-1}\left(\mathcal{I}, \mathbb{R}^{m}\right)$, a finite-dimensional solution space for the homogeneous DAE as well as an inherent regular ODE that determines the dynamics of the DAE.

Definition 2.4 An equation (2.1) with properly stated leading term is said to be a regular index $\mu D A E$ on the interval $\mathcal{I}, \mu \in \mathbb{N}$, if there is a continuous matrix function sequence (2.2) such that

(a) $G_{i}(t)$ has constant rank $r_{i} \geq 0$ on $\mathcal{I}, i \geq 0$,

(b) condition (2.7) is satisfied,

(c) $Q_{i} \in C\left(\mathcal{I}, L\left(\mathbb{R}^{m}\right)\right), D P_{0} \cdots P_{i} D^{-} \in C^{1}\left(\mathcal{I}, \mathbb{R}^{n}\right), i \geq 0$,

(d) $0 \leq r_{0} \leq \cdots \leq r_{\mu-1}<m$ and $r_{\mu}=m$.

The DAE (2.1) is called regular if it is regular with some index $\mu$.

Not surprisingly, by Theorem 2.3 constant coefficient DAEs are regular with index $\mu$ if and only if the pair $\{A D, B\}$ forms a regular matrix pencil with index $\mu$.

At this place it has to be mentioned that in the literature concerning variable coefficient (standard form) DAEs, the word "regular" is often used and with quite different meanings.

Example 2.1 The DEA (2.1) given by the coefficients

$$
A(t)=\left(\begin{array}{cc}
1 & 0 \\
-t & 1 \\
0 & 0
\end{array}\right), \quad D(t)=\left(\begin{array}{lll}
0 & 1 & 0 \\
0 & 0 & 1
\end{array}\right), B(t)=\left(\begin{array}{ccc}
1 & 0 & 0 \\
0 & 0 & 0 \\
0 & -t & 1
\end{array}\right), \quad t \in \mathcal{I}=\mathbb{R},
$$

reads in detail

$$
x_{2}^{\prime}+x_{1}=q_{1}, \quad-t x_{2}^{\prime}+x_{3}^{\prime}=q_{2}, \quad-t x_{2}+x_{3}=q_{3} .
$$

This DAE is regular with index 3 in the sense of Defintion 2.4. Namely, we derive here

$$
\begin{aligned}
R(t)=\left(\begin{array}{ll}
1 & 0 \\
0 & 1
\end{array}\right), & \left(\begin{array}{ccc}
0 & 1 & 0 \\
0 & -t & 1 \\
0 & 0 & 0
\end{array}\right), Q_{0}(t)=\left(\begin{array}{lll}
1 & 0 & 0 \\
0 & 0 & 0 \\
0 & 0 & 0
\end{array}\right), D(t)^{-}=\left(\begin{array}{ll}
0 & 0 \\
1 & 0 \\
0 & 1
\end{array}\right), \\
G_{0}(t) & \\
G_{1}(t) & =\left(\begin{array}{ccc}
1 & 1 & 0 \\
0 & -t & 1 \\
0 & 0 & 0
\end{array}\right), Q_{1}(t)=\left(\begin{array}{ccc}
0 & -1 & 0 \\
0 & 1 & 0 \\
0 & t & 0
\end{array}\right), G_{2}(t)=\left(\begin{array}{ccc}
1 & 1 & 0 \\
0 & 1-t & 1 \\
0 & 0 & 0
\end{array}\right),
\end{aligned}
$$




$$
Q_{2}(t)=\left(\begin{array}{ccc}
0 & -t & 1 \\
0 & t & -1 \\
0 & -t(1-t) & 1-t
\end{array}\right), G_{3}(t)=\left(\begin{array}{ccc}
1 & 1 & 0 \\
0 & 1-t & 1 \\
0 & -t & 1
\end{array}\right), \operatorname{det} G_{3}(t)=1
$$

thus $m=2, n=2, \quad r_{0}=r_{1}=r_{2}=2, \quad r_{3}=3, D(t) P_{1}(t) D(t)^{-}=\left(\begin{array}{cc}0 & 0 \\ -t & 1\end{array}\right)$, $D(t) Q_{1}(t) D(t)^{-}=\left(\begin{array}{ll}1 & 0 \\ t & 0\end{array}\right), D(t) P_{1}(t) P_{2}(t) D(t)^{-}=\left(\begin{array}{ll}0 & 0 \\ 0 & 0\end{array}\right)$.

Here, the inherent regular ODE disappears (cf. Section 3 ) and $q=0$ implies $x=0$, i.e., the homogeneous equation has only the trivial solution. Notice that the condition ker $G_{0}(t) \cap \operatorname{ker} B(t)=0$ is valid but it holds that $\operatorname{det}\left(\lambda G_{0}(t)+B(t)\right)=0$ for all $t$ and $\lambda$, i.e., the matrix pencil $\{A(t) D(t), B(t)\}$ is singular on $\mathcal{I}$. Because of this singular local pencil, this DAE rewritten in standard form $A D x^{\prime}+B x=q$ fails to be a regular DAE in the sense of $[\mathrm{BrCaPe}]$ and the coefficient pair $\{A() D.(),. B()$.$\} is not a regular$ matrix pair in the sense of [Bo]. Nevertheless, (2.8) is easily checked to have differentiation index 3 .

Example 2.2: The DAE (2.1) given by the coefficients $A(t)=\left(\begin{array}{c}t \\ 1\end{array}\right), D(t)=\left(\begin{array}{ll}-1 & t\end{array}\right)$, $B(t)=\left(\begin{array}{cc}1 & -t \\ 0 & 0\end{array}\right), t \in \mathcal{I}=\mathbb{R}$ yields $G_{0}(t)=\left(\begin{array}{cc}-t & t^{2} \\ -1 & t\end{array}\right), \quad N_{0}(t) \cap \operatorname{ker} B(t)=N_{0}(t)$, thus $G_{1}(t)=G_{0}(t), N_{0}(t)=N_{1}(t)$ independently of the choice of $Q_{0}(t)$. This is no more a regular DAE in the sense of our Definition 2.4. By simple inserting it can be verified that all functions $x(t)=\gamma(t)\left(\begin{array}{c}t \\ 1\end{array}\right), t \in \mathcal{I}$, where $\gamma \in C(\mathcal{I}, \mathbb{R})$ is completely arbitrary, satisfy the corresponding homogeneous equation.

Rewritten in standard form $A D x^{\prime}+\left(B+A D^{\prime}\right) x=0$, this DAE is in detail

$$
\left(\begin{array}{cc}
-t & t^{2} \\
-1 & t
\end{array}\right) x^{\prime}(t)+x(t)=0
$$

Because of $\operatorname{ker}(A(t) D(t))^{*} \cap \operatorname{ker}\left(B(t)+A(t) D^{\prime}(t)\right)^{*}=0$ for $t \in \mathcal{I}$ in the context of [RaRh], this special coefficient pair $\left\{A D, B+A D^{\prime}\right\}$ is a regular one, but is it no more completely regular. The DAE (2.9) itself is reducible but no more completely reducible in the sense of [RaRh]. Notice that the local pencil of (2.9) is regular for all $t \in \mathcal{I}$ such that this DAE is said to be regular in [CaBrPe] and its coefficients form a regular pair in the sense of $[\mathrm{Bo}]$.

If the projectors $D P_{1} \cdots P_{i} D^{-}, i \geq 1$, are just time-invariant, the corresponding derivative terms in the matrix function sequence (2.2) disappear and the expression for $B_{i}$ simplifies to $B_{i}=B_{i-1} P_{i-1}$. Then, if there is a $\mu \in I N$ such that $G_{\mu}(t)$ is nonsingular but $G_{\mu-1}(t)$ is singular, by Theorem 2.3, the pair $\{A(t) D(t), B(t)\}$ forms a regular index $\mu$ pencil. This leads to the next proposition.

Proposition 2.5 If (2.1) is a regular index $\mu D A E$ on $\mathcal{I}$ and if it holds that $\left(D P_{1} \ldots\right.$ $\left.P_{i} D^{-}\right)^{\prime}=0$ for $i=1, \ldots \mu-1$, then, for each $t \in \mathcal{I}$, the local matrix pair $\{A(t) D(t), B(t)\}$ forms a pencil that is regular with index $\mu$. 
Clearly, in case of a regular index 1 DAE (2.1), the pair $\{A(t) D(t), B(t)\}$ forms a regular index 1 pencil uniformly for all $t \in \mathcal{I}$.

Remark 2.6 Our index notion generalizes the constant coefficient Kornecker index (cf. Theorem 2.3) and the tractability index (e.g. [Mä1], [Ha], [Mä2]) given for standard DAEs

$$
E(t) x^{\prime}(t)+F(t) x(t)=q(t), \quad t \in I,
$$

via the reformulation with properly stated leading term

$$
E(t)\left(P_{E}(t) x(t)\right)^{\prime}+\left(F(t)-E(t) P_{E}^{\prime}(t)\right) x(t)=q(t), \quad t \in I,
$$

by means of a continuoulsy differentiable projector function $P_{E}(t)$ with $k e r P_{E}=k e r E$. In (2.11), thought as (2.1), $P_{E}$ plays the roles of $D, R, D^{-}$and $P_{0}$ simultaneously. We have $G_{0}=E, B_{-1}=F-E P_{E}^{\prime}$, and for $i \geq 0$,

$$
\begin{aligned}
G_{i+1} & =G_{i}+B_{i-1} P_{i-1} Q_{i}-G_{i} P_{0}\left(P_{0} \cdots P_{i}\right)^{\prime} P_{0} \cdots P_{i-1} Q_{i} \\
& =G_{i}+B_{i-1} P_{i-1} Q_{i}-G_{i}\left(P_{0} \cdots P_{i}\right)^{\prime} P_{0} \cdots P_{i-1} Q_{i}
\end{aligned}
$$

i.e., we obtain precisely the matrices used to define the tractability index as well as to prove corresponding solvability statements.

Remark 2.7 DAEs with properly stated leading terms of tractability index 1 and index 2 in the sense of [BaMä] are now called regular DAEs of index 1 or 2, respectively. Namely, it holds that $N_{0}(t) \cap S_{0}(t)=0$ if and only if $G_{1}(t)$ is nonsingular. $G_{1}(t)$ has a constant rank on $I$ if and only if $N_{0}(t) \cap S_{0}(t)$ does not change its dimension and, finally, $G_{2}(t)$ is nonsingular if and only if $N_{1}(t) \cap S_{1}(t)=0$. Further, $N_{1}(t) \cap S_{1}(t)=0$ implies ([GrMä1], Theorem A. 13) $N_{1}(t) \oplus S_{1}(t)=\mathbb{R}^{m}$, and it holds that $N_{0} \subset S_{1}$, thus $N_{1} \cap N_{0}=0$.

A regular index-1 DAE can be equivalently characterized by $N_{0}(t) \cap S_{0}(t)=0, t \in I$. A regular index-2 DAE can be characterized by $\operatorname{dim}\left(N_{0}(t) \cap S_{0}(t)\right)=$ const $>0, N_{1}(t) \cap$ $S_{1}(t)=0 t \in I$, which is done in [BaMä].

Remark 2.8 A DAE in so-called strong standard canonical form (SSCF) consists of the two decoupled systems ([CaPe])

$$
x_{1}^{\prime}(t)+W(t) x_{1}(t)=q_{1}(t), \quad N x_{2}^{\prime}(t)+x_{2}(t)=q_{2}(t)
$$

with a constant nilpotent matrix $N$. In this special case one obtains a fully constant matrix sequence $\left\{G_{i}\right\}_{i \geq 0}$, namely

$G_{0}=\left(\begin{array}{cc}I & 0 \\ 0 & N\end{array}\right), \quad Q_{0}=\left(\begin{array}{cc}0 & 0 \\ 0 & Q_{N, 0}\end{array}\right), \quad G_{1}=\left(\begin{array}{cc}I & 0 \\ 0 & N+Q_{N, 0}\end{array}\right), \quad Q_{1}=\left(\begin{array}{cc}0 & 0 \\ 0 & Q_{N, 1}\end{array}\right)$

and so on. In the lower right corners, the sequence corresponding to the constant matrix pair $\{N, I\}$ arises. Thus, the SSCF-DAE (2.12) rewritten with properly stated leading term (e.g. with $N x_{2}^{\prime}$ replaced by $\left.N\left(P_{N, 0} x_{2}(t)\right)^{\prime}\right)$ is regular with index $\mu=\operatorname{ind}(N)$. 
Remark 2.9 If $G_{0}(t)$ remains nonsingular except for a certain point $t_{*} \in \mathcal{I}$, we usually speak of an ODE that has singularity at $t_{*}$. Similarly, if $G_{0}(t)$ is singular, we treat points at which the constant rank condition fails as exceptional ones and call them singularities. Further singularities arise if the rank of $G_{i}(t), i>0$, changes. This understanding shares the view taken in [RaRh] and [KuMe]. A precise description of possible singularities lies ahead. In particular, if the constant matrix $N$ in (2.12) is replaced by a time-varying, strictly upper triangular $N(t)$, the resulting system is said to be (cf. [CaPe]) a DAE in standard canonical form (SCF). It is well known ([Ca], [CaPe]) that singularities caused by rank changes in $N(t)$ are somehow harmless (cf. Example 5.1 below). This interesting feature and its consequences for numerical methods are worth being considered in more detail.

By construction, the matrix functions $G_{i}$ determined by (2.2) depend on how the projector functions $Q_{j}$ are chosen. Hence, the question arises whether regularity and the index of a DAE (2.1) depend on the special choice of the projectors. However, this is not the case. In order to realize this fact, we take two different continuous projector functions $Q_{0}$ and $\tilde{Q}_{0}$ onto $N_{0}=\operatorname{ker} G_{0}$, and build up the two sequences. Denote by $D^{-}$and $\tilde{D}^{-}$the corresponding reflexive generalized inverses of $D$. It holds that $\tilde{D}^{-}=\tilde{P}_{0} D^{-}, D^{-}=P_{0} \tilde{D}^{-}$. Derive $\tilde{G}_{1}=G_{0}+B \tilde{Q}_{0}=G_{0}+B \tilde{Q}_{0} Q_{0}+B \tilde{Q}_{0} P_{0}=$ $G_{0}+B Q_{0}+B \tilde{Q}_{0} P_{0}=\left(G_{0}+B Q_{0}\right)\left(I+Q_{0} \tilde{Q}_{0} P_{0}\right)=G_{1}\left(I+Q_{0} \tilde{Q}_{0} P_{0}\right)$. Since the factor $E_{1}=I+Q_{0} \tilde{Q}_{0} P_{0}$ is nonsingular, $\tilde{G}_{1}$ and $G_{1}$ have the same rank. $Q_{1}$ is a continuous projector function onto $N_{1}$ at the same time as $\tilde{Q}_{1}=E_{1} Q_{1} E_{1}^{-1}=E_{1} Q_{1}\left(I-Q_{0} \tilde{Q}_{0} P_{0}\right)$ projects onto $\tilde{N}_{1}$ and is continuous. Because of $\tilde{Q}_{1} \tilde{Q}_{0}=E_{1} Q_{1}\left(I-Q_{0} \tilde{Q}_{0} P_{0}\right) \tilde{Q}_{0}=$ $E_{1} Q_{1} \tilde{Q}_{0}=E_{1} Q_{1} Q_{0} \tilde{Q}_{0}$, the relation $Q_{1} Q_{0}=0$ implies $\tilde{Q}_{1} \tilde{Q}_{0}=0$ and vice versa.

It comes out that, at this stage, both sequences satisfy (2.7) or both do not. If they do, it holds that $D \tilde{P}_{0} \tilde{P}_{1} \tilde{D}^{-}=D P_{0} P_{1} D^{-}$, i.e., the additional smoothness transfers from the first sequence to the second one and vice versa.

Next, by induction, the expression $\tilde{G}_{i+1}=G_{i+1} E_{i+1}$ with certain continuous nonsingular factors $E_{i+1}=I+Q_{0} \mathfrak{B}_{i+1} P_{0}$ can be obtained, where projector functions $\tilde{Q}_{j}=$ $E_{j} Q_{j} E_{j}^{-1}$ are used for $1 \leq j \leq i$. Due to the relations $\tilde{Q}_{j} \tilde{Q}_{k}=E_{j} Q_{j} Q_{k} E_{k}^{-1}, 0 \leq k<j$, condition (2.7) is given at the actual stage for both sequences or it fails for both of them. If (2.7) is satisfied, it holds that $D \tilde{P}_{0} \cdots \tilde{P}_{j} \tilde{D}^{-}=D P_{0} \cdots P_{j} D^{-}$, and so on.

Proposition 2.10 Regularity with index $\mu$ does not depend on the choice of the involved in the matrix function sequence projectors.

Proof: Above, we have compared two matrix function sequences which have two different projector functions $Q_{0}$ and $\tilde{Q}_{0}$ to start with. It remains to check that nothing worse will happen if we continue a given matrix function chain $G_{0}, \ldots G_{i}$ satisfying the conditions (a), (b) and (c) up to this stage $i>0$ by means of two different projector functions $Q_{i}$ and $\tilde{Q}_{i}$. Comparing the resulting two matrix function sequences is quite similar to the case of two starting projector functions. Because of the technical amount we have placed this part in the Appendix.

By Proposition 2.10, the definition of DAEs (2.1) being regular with index $\mu$ is confirmed to be reasonable. 


\section{Inherent regular ODEs}

As described in [BaMä], a regular DAE

$$
A(D x)^{\prime}+B x=q
$$

with index 2 can be decoupled into

$$
x=K_{2} D^{-} u+\left(P_{0} Q_{1}+Q_{0} P_{1}\right) G_{2}^{-1} q+Q_{0} Q_{1} D^{-}\left(D Q_{1} G_{2}^{-1} q\right)^{\prime}
$$

where $u \in C^{1}\left(I, \mathbb{R}^{n}\right)$ satisfies the inherent regular ODE

$$
u^{\prime}-\left(D P_{1} D^{-}\right)^{\prime} u+D P_{1} G_{2}^{-1} B D^{-} u=D P_{1} G_{2}^{-1} q .
$$

Thereby, $Q_{1}$ is taken as the canonical projector onto $N_{1}$ along $S_{1}$. The matrix function

$$
K_{2}=I-Q_{0} Q_{1} D^{-}\left(D Q_{1} D^{-}\right)^{\prime} D-Q_{0} P_{1} G_{2}^{-1} B P_{0}
$$

is nonsingular. One could also define $Q_{0}$ in a special canonical way that would lead to $K_{2}=I$. However, working with an arbitrary $Q_{0}$ seems to be more comfortable. In any case, the coefficients of the inherent regular ODE (3.3), but also $D Q_{1} G_{2}^{-1}$ are independent of the choice of $Q_{0}$ (cf. [BaMä]). We will dwell upon the case of $\mu=2$ for a moment.

For each solution $x \in C_{D}^{1}\left(I, \mathbb{R}^{m}\right)$ of (3.1) we obtain $D Q_{1} G_{2}^{-1} q=D Q_{1} x=D Q_{1} D^{-} D x \in$ $C^{1}\left(I, \mathbb{R}^{n}\right)$ as well as the representation (3.2), (3.3) with $u=D P_{1} x$. Conversely, if $u \in C^{1}\left(I, \mathbb{R}^{n}\right)$ satisfies (3.3) and, additionally, the initial condition $u\left(t_{0}\right)=u_{0} \in$ im $D\left(t_{0}\right) P_{1}\left(t_{0}\right)$, then $u(t)$ remains in $i m D(t) P_{1}(t)$ for all $t \in I$, and, provided that $D Q_{1} G_{2}^{-1} q \in C^{1}\left(I, \mathbb{R}^{n}\right)$, the function $x$ resulting from (3.2) is a solution of (3.1) and satisfies the initial condition $D\left(t_{0}\right) P_{1}\left(t_{0}\right) x\left(t_{0}\right)=u_{0}$. Moreover, it holds that $D P_{1} x=u$. The inherent regular ODE (3.3) has the time-varying subspace im $D(t) P_{1}(t) D(t)^{-}=$ im $D(t) P_{1}(t)=D(t) S_{1}(t)$ as an invariant subspace, i.e., if a solution belongs to $D\left(t_{0}\right) S_{1}\left(t_{0}\right)$ at a certain $t_{0} \in I$, it lies in $D(t) S_{1}(t)$ for all $t \in I$. Due to the relation $D P_{1} x=u$ we are exclusively interested in those solutions of the inherent regular ODE that belong to this basic invariant subspace.

In this way, the dynamics of (3.1) is dominated by the flow of the inherent regular ODE (3.3) along its basic invariant subspace $D S_{1}=i m D P_{1} D^{-}$.

For the index-2 DAE (3.1) the dynamical degree of freedom is given by $\operatorname{dim} D(t) S_{1}(t)=$ $\operatorname{rank} D(t) P_{1}(t) D(t)^{-}=m-\operatorname{rank} G_{1}(t)$.

If $D(t) S_{1}(t)$ actually does not vary with $t$, one can turn to minimal coordinates and use $D(t) S_{1}(t)$ as the (constant) state space.

In case of $\mu>2$, the situation is similar. However, the technical amount is much greater. Here, we do not aim at complete solution representations as given for $\mu=2$ by (3.2), and corresponding sharp solvability statements. We direct our interest to the inherent regular ODE.

Theorem 3.1 For each solution $x \in C_{D}^{1}\left(I, \mathbb{R}^{m}\right)$ of a regular index $\mu D A E$ (3.1), the component $D P_{0} \cdots P_{\mu-1} D^{-} D x=D P_{0} \cdots P_{\mu-1} x=: u \in C^{1}\left(I, \mathbb{R}^{n}\right)$ satisfies the inherent regular $O D E$

$$
u^{\prime}-\left(D P_{0} \cdots P_{\mu-1} D^{-}\right)^{\prime} u+D P_{0} \cdots P_{\mu-1} G_{\mu}^{-1} B D^{-} u=D P_{0} \cdots P_{\mu-1} G_{\mu}^{-1} q .
$$


The time varying subspace im $D P_{0} \cdots P_{\mu-1} D^{-}$is an invariant subspace of the inherent regular ODE (3.4).

Proof: We premultiply (3.1) by $G_{\mu}^{-1}$ and take the following relations into account:

$$
\begin{aligned}
& G_{\mu}^{-1} A(D x)^{\prime}=G_{\mu}^{-1} A D D^{-}(D x)^{\prime}=G_{\mu}^{-1} G_{\mu} P_{\mu-1} \cdots P_{0} D^{-}(D x)^{\prime}, \\
& B=B_{0}, \\
& G_{\mu}^{-1} B=G_{\mu}^{-1} B P_{0} \cdots P_{\mu-1}+G_{\mu}^{-1} B_{0} P_{0} \cdots P_{\mu-2} Q_{\mu-1}+\cdots+G_{\mu}^{-1} B_{0} P_{0} Q_{1}+G_{\mu}^{-1} B_{0} Q_{0} \\
& \text { and } \\
& B_{0} Q_{0}=G_{1} Q_{0}=G_{\mu} P_{\mu-1} \cdots P_{1} Q_{0}=G_{\mu} Q_{0}, \\
& B_{0} P_{0} \cdots P_{i-1} Q_{i}=B_{i} Q_{i}+\sum_{j=1}^{i} G_{j} D^{-}\left(D P_{0} \cdots P_{j} D^{-}\right)^{\prime} D P_{0} \cdots P_{i-1} Q_{i} \\
& \quad=G_{\mu} Q_{i}+\sum_{j=1}^{i} G_{\mu} P_{\mu-1} \cdots P_{j} D^{-}\left(D P_{0} \cdots P_{j} D^{-}\right)^{\prime} D P_{0} \cdots P_{i-1} Q_{i},
\end{aligned}
$$

for $i=1, \ldots \mu-1$.

Consequently, the equation (3.1) scaled by $G_{\mu}^{-1}$ reads

$$
\begin{aligned}
P_{\mu-1} \cdots P_{0} D^{-}(D x)^{\prime} & +G_{\mu}^{-1} B P_{0} \cdots P_{\mu-1} x+Q_{\mu-1} x+\cdots+Q_{0} x \\
& +\sum_{i=1}^{\mu-1} \sum_{j=1}^{i} P_{\mu-1} \cdots P_{j} D^{-}\left(D P_{0} \cdots P_{j} D^{-}\right)^{\prime} D P_{0} \cdots P_{i-1} Q_{i} x=G_{\mu}^{-1} q .
\end{aligned}
$$

Multiplying (3.5) by $D P_{0} \cdots P_{\mu-1}$ and taking into account that

$$
\begin{aligned}
& D P_{0} \cdots P_{\mu-1} P_{\mu-1} \cdots P_{0} D^{-}(D x)^{\prime}=D P_{0} \cdots P_{\mu-1} D^{-}(D x)^{\prime}= \\
& \left(D P_{0} \cdots P_{\mu-1} x\right)^{\prime}-\left(D P_{0} \cdots P_{\mu-1} D^{-}\right)^{\prime} D x
\end{aligned}
$$

and

$$
\begin{aligned}
& \sum_{i=1}^{\mu-1} \sum_{j=1}^{i} D P_{0} \cdots P_{\mu-1} P_{\mu-1} \cdots P_{j} D^{-}\left(D P_{0} \cdots P_{j} D^{-}\right)^{\prime} D P_{0} \cdots P_{i-1} Q_{i} \\
& =\sum_{i=1}^{\mu-1} \sum_{j=1}^{i} D P_{0} \cdots P_{\mu-1} D^{-}\left(D P_{0} \cdots P_{j} D^{-}\right)^{\prime} D P_{0} \cdots P_{i-1} Q_{i} \\
& =\sum_{i=1}^{\mu-1} \sum_{j=1}^{i}\left\{\left(D P_{0} \cdots P_{\mu-1} D^{-}\right)^{\prime} D P_{0} \cdots P_{i-1} Q_{i}-\right. \\
& \left.\quad-\left(D P_{0} \cdots P_{\mu-1} D^{-}\right)^{\prime} D P_{0} \cdots P_{j} D^{-} D P_{0} \cdots P_{i-1} Q_{i}\right\} \\
& =\sum_{i=1}^{\mu-1}\left(D P_{0} \cdots P_{\mu-1} D^{-}\right)^{\prime}\left\{i D P_{0} \cdots P_{i-1} Q_{i}-\sum_{j=1}^{i-1} D P_{0} \cdots P_{j} P_{0} \cdots P_{i-1} Q_{i}\right\} \\
& =\sum_{i=1}^{\mu-1}\left(D P_{0} \cdots P_{\mu-1} D^{-}\right)^{\prime} D P_{0} \cdots P_{i-1} Q_{i}
\end{aligned}
$$

we obtain

$$
\begin{aligned}
\left(D P_{0} \cdots P_{\mu-1} x\right)^{\prime} & -\left(D P_{0} \cdots P_{\mu-1} D^{-}\right)^{\prime}\left\{D x-\sum_{i=1}^{\mu-1} D P_{0} \cdots P_{i-1} Q_{i}\right\} \\
& +D P_{0} \cdots P_{\mu-1} G_{\mu}^{-1} B D^{-} D P_{0} \cdots P_{\mu-1} x=D P_{0} \cdots P_{\mu-1} G_{\mu}^{-1} q
\end{aligned}
$$


but this leads immediately to the inherent regular ODE (3.4).

It remains to check that $i m D P_{0} \cdots P_{\mu-1}$ is actually an invariant subspace of the ODE (3.4).

Supposed $u \in C^{1}\left(I, \mathbb{R}^{n}\right)$ satisfies (3.4) and $t_{0} \in I, u\left(t_{0}\right)=u_{0} \in \mathrm{im} D\left(t_{0}\right) P_{0}\left(t_{0}\right) \cdots$ $P_{\mu-1}\left(t_{0}\right)$. Multiplying the resulting identity $(3.5)$ by $\left(I-D P_{0} \cdots P_{\mu-1} D^{-}\right)$yields

$$
\left(I-D P_{0} \cdots P_{\mu-1} D^{-}\right) u^{\prime}-\left(I-D P_{0} \cdots P_{\mu-1} D^{-}\right)\left(D P_{0} \cdots P_{\mu-1} D^{-}\right)^{\prime} u=0
$$

and for $\alpha:=\left(I-D P_{0} \cdots P_{\mu-1} D^{-}\right) u$,

$$
\alpha^{\prime}+\left(D P_{0} \cdots P_{\mu-1} D^{-}\right)^{\prime} \alpha=0
$$

Because of $\alpha\left(t_{0}\right)=0$ it follows that $\alpha$ vanishes identically, i.e., $u=D P_{0} \cdots P_{\mu-1} D^{-} u$.

Remark 3.2 The subspace $i m D P_{0} \cdots P_{\mu-1}=i m D P_{0} \cdots P_{\mu-1} D^{-}$is said to be the basic invariant subspace of the inherent regular ODE (3.4).

Stress once more that only those solutions of the inherent regular ODE (3.4) that lie in the basic invariant subspace are relevant for the DAE. Even if this basic invariant subspace actually varies with $t$, we know the dynamical degree of freedom to be equal to $\sum_{i=0}^{\mu-1} \operatorname{rank} G_{i}-(\mu-1) m=: d_{\mu}$.

If the $d_{\mu}$-dimensional basic invariant subspace $i m D P_{0} \cdots P_{\mu-1} D^{-}$is just time-invariant, one can take advantage of a constant state space and apply standard results on explicit ODEs (cf. [HiMäTi] for consequences in view of numerical integration methods). As we shall see below, the basic invariant subspace changes under refactorizations of the leading term, i.e., refactorizations can be understood as a tool for transforming the actual dynamic component.

\section{Invariance under transformations and refactor- izations}

We continue investigating the linear continuous coefficient DAE

$$
A(D x)^{\prime}+B x=q
$$

with properly stated leading term. By means of any nonsingular matrix functions

$$
K, L \in C\left(I, L\left(\mathbb{R}^{m}\right)\right),
$$

we change the variable $x=K \tilde{x}$ and scale the equation (4.1) such that a new DAE

$$
\tilde{A}(\tilde{D} \tilde{x})^{\prime}+\tilde{B} \tilde{x}=L q
$$

results, which has again continuous coefficients

$$
\tilde{A}=L A, \quad \tilde{D}=D K, \quad \tilde{B}=L B K .
$$

Obviously, the leading term of (4.3) is properly stated since $A$ and $D$ are well matched. $R$ and $\tilde{R}$ coincide. 
If $x \in C_{D}^{1}\left(I, \mathbb{R}^{m}\right)$ is a solution of the $\operatorname{DAE}(4.1)$, then $\tilde{x}=K^{-1} x \in C_{\tilde{D}}^{1}\left(I, \mathbb{R}^{m}\right)$ solves the transformed DAE (4.3), and vice versa.

Next we compare the sequences of matrices for both the original and the transformed DAEs and check whether regularity with index $\mu$ is invariant.

We have $G_{0}=A D, B_{-1}=B, B_{0}=B$, but $\tilde{G}_{0}=\tilde{A} \tilde{D}=L G_{0} K, \tilde{B}_{-1}=L B K, \tilde{B}_{0}=$ $K B_{0} K$.

Letting $\tilde{Q}_{0}=K^{-1} Q_{0} K$, we derive $\tilde{D}^{-}=K^{-1} D^{-}, \tilde{G}_{1}=\tilde{G}_{0}+\tilde{B}_{0} \tilde{Q}_{0}=L\left(G_{0}+\right.$ $\left.B_{0} Q_{0}\right) K=L G_{0} K$. Further, with $\tilde{Q}_{1}=K^{-1} Q_{1} K$ we compute

$$
\begin{aligned}
\tilde{Q}_{1} \tilde{Q}_{0} & =K^{-1} Q_{1} Q_{0} K \\
\tilde{D} \tilde{P}_{0} \tilde{P}_{1} \tilde{D}^{-} & =D P_{0} P_{1} D^{-} \\
\tilde{G}_{2} & =\tilde{G}_{1}+\tilde{B}_{0} \tilde{P}_{0} \tilde{Q}_{1}-\tilde{G}_{1} \tilde{D}^{-}\left(\tilde{D} \tilde{P}_{0} \tilde{P}_{1} \tilde{D}^{-}\right)^{\prime} \tilde{D} \tilde{Q}_{1} \\
& =L\left(G_{1}+B_{0} Q_{0}-G_{1}\left(D P_{0} P_{1} D^{-}\right)^{\prime} Q_{1}\right) K \\
& =L G_{2} K
\end{aligned}
$$

and so on, $\tilde{G}_{i}=L G_{i} K, i \geq 2$.

In particular, the property (2.7) is transformed from (4.1) to (4.3). Due to

$$
\tilde{D} \tilde{P}_{0} \cdots \tilde{P}_{i} \tilde{D}^{-}=D P_{0} \cdots P_{i} D^{-}, \quad i \geq 0
$$

the basic smoothness is also transferred. More precisely, the subspaces corresponding to (4.5) are not touched at all by the transformations.

Theorem 4.1 If the DAE (4.1) is regular with index $\mu$, then the transformed DAE (4.3) is also regular with index $\mu$.

Both index $\mu$ DAEs have a common inherent regular ODE as well as a common basic invariant subspace.

Due to Theorem 4.1, the equations (1.4), (1.5), (1.7) and (1.8) in Section 1 have altogether the index $\mu=$ ind $(E, F)$.

Besides the premultiplication and the change of variables, we consider also refactorizations of the leading term. As mentioned above, a refactorization corresponds somehow to a transformation of the inner vector field. We shall see below how a refactorization changes the inherent regular ODEs as well as the basic invariant subspaces.

Recall the equations (1.7) and (1.9) to differ just by such a refactorization.

Now take an arbitrary nonsingular matrix function

$$
H \in C^{1}\left(I, L\left(\mathbb{R}^{n}\right)\right)
$$

and rewrite (4.1) by means of

$$
A(D x)^{\prime}=A\left(H H^{-1} D x\right)^{\prime}=A H\left(H^{-1} D x\right)^{\prime}+A H^{\prime} H^{-1} D x
$$

as

$$
\tilde{A}(\tilde{D} x)^{\prime}+\tilde{B} x=q,
$$


where

$$
\tilde{A}=A H, \quad \tilde{D}=H^{-1} D, \quad \tilde{B}=B+A D D^{-} H^{\prime} H^{-1} D .
$$

For trivial reasons, $\tilde{A}$ and $\tilde{D}$ are well matched at the same time as $A$ and $D$ are so. It results that $\tilde{R}=H^{-1} R H$. The solution spaces for the original DAE and the refactorized one coincide, i.e., $C_{D}^{1}\left(I, \mathbb{R}^{m}\right)=C_{\tilde{D}}^{1}\left(I, \mathbb{R}^{m}\right)$.

Next, we deal with the question whether these DAEs may have a different index. For this purpose we compare the corresponding sequences of matrix functions.

Since $G_{0}=A D=\tilde{A} \tilde{D}=\tilde{G}_{0}$, we may put $\tilde{Q}=Q_{0}, \tilde{D}^{-}=D^{-} H$. We have $\tilde{G}_{1}=$ $G_{0}+\tilde{B} Q_{0}=G_{0}+B Q_{0}=G_{1}$, thus $\tilde{W}_{1}=W_{1}, \tilde{Q}_{1}=Q_{1}$. However, in the next step we obtain $\tilde{G}_{2}=G_{1}+\tilde{B}_{1} Q_{1}=G_{1}+B_{1} Q_{1}+G_{0} Q_{1} D^{-} H^{\prime} H^{-1} D Q_{1}$, hence,

$$
\tilde{G}_{2}=G_{2}\left(I+P_{1} P_{0} Q_{1} D^{-} H^{\prime} H^{-1} D Q_{1}\right) \text {. }
$$

The factor

$$
F_{2}=I+P_{1} P_{0} Q_{1} D^{-} H^{\prime} H^{-1} D Q_{1}=I-Q_{0} Q_{1} D^{-} H^{\prime} H^{-1} D Q_{1}
$$

is always nonsingular.

Lemma 4.2 Set $F_{0}=I, F_{1}=I$. With nonsingular factors

$$
F_{k}=I-\sum_{l=0}^{k-2} Q_{l} \mathfrak{A}_{k l} Q_{k-1}, \quad k \geq 2,
$$

where the $\mathfrak{A}_{k l}$ denote certain matrix functions, and with projectors $\tilde{Q}_{j}=\left(F_{j} \cdots F_{0}\right)^{-1} Q_{j} F_{j} \cdots F_{0}, j=0, \cdots, i-1$, it holds that

$$
\tilde{G}_{i}=G_{i} F_{i} \cdots F_{0} \text { for all } i \geq 0 \text {. }
$$

$\mathbf{P} \mathbf{r}$ o o f : The relation (4.11) is trivial for $i=0$ and $i=1$, but it holds for $i=2$ by (4.9). It will be verified for $i \geq 3$ by a straigtforward but technically expensive induction in the Appendix.

Due to the special structure of the factors $F_{k}$ the following properties may be used:

$$
\begin{aligned}
& F_{k}^{-1}=I+Q_{k-2} \mathfrak{A}_{k k-2} Q_{k-1}+\cdots+Q_{0} \mathfrak{A}_{k 0} Q_{k-1}, \\
& F_{k} Q_{j}=Q_{j}=F_{k}^{-1} Q_{j}, \quad j=0, \ldots, k-2, \\
& Q_{i} F_{k}=Q_{i}=Q_{i} F_{k}^{-1}, \quad i \geq k-1, \\
& P_{0} \cdots P_{i} F_{j}=P_{0} \cdots P_{i}=P_{0} \cdots P_{i} F_{j}^{-1}, \quad i \geq j-2 .
\end{aligned}
$$

The matrices $G_{i}$ and $\tilde{G}_{i}$ have always the same rank.

Set $\tilde{Q}_{i}=\left(F_{i} \cdots F_{0}\right)^{-1} Q_{i} F_{i} \cdots F_{0}=\left(F_{i} \cdots F_{0}\right)^{-1} Q_{i}, \quad i \geq 0$, so that for $j \leq i-1$,

$$
\tilde{Q}_{i} \tilde{Q}_{j}=\left(F_{i} \cdots F_{0}\right)^{-1} Q_{i}\left(F_{i} \cdots F_{0}\right)^{-1} Q_{j}=\left(F_{i} \cdots F_{0}\right)^{-1} Q_{i} Q_{j}=0,
$$


i.e., the property (2.7) transfers from the original DAE (4.1) to (4.7). Moreover, since

$$
\begin{aligned}
\tilde{P}_{0} \cdots \tilde{P}_{i} & =F_{0}^{-1} P_{0} F_{0}\left(F_{1} F_{0}\right)^{-1} P_{1} F_{1} P_{0} \cdots\left(F_{i} \cdots F_{0}\right)^{-1} P_{i} F_{i} \cdots P_{0} \\
& =F_{0}^{-1} P_{0} F_{1}^{-1} P_{1} \cdots F_{i}^{-1} P_{i} F_{i} \cdots P_{0}=P_{0} \cdots P_{i} F_{i} \cdots P_{0} \\
& =P_{0} \cdots P_{i}, \quad i \geq 0,
\end{aligned}
$$

the subspaces determined by these projectors do not change. However, it turns out that

$$
\tilde{D} \tilde{P}_{0} \cdots \tilde{P}_{i} \tilde{D}^{-}=H^{-1} D P_{0} \cdots P_{i} D^{-} H
$$

hence, the basic smoothness is maintained, but the corresponding projectors are subject to a similarity transformation.

Theorem 4.3 Let (4.1) be a regular DAE with index $\mu$.

(i) Then, the refactorized DAE (4.7) is also regular with index $\mu$.

(ii) The resulting inherent regular $O D E$ of the DAE (4.7) coincides along its basic invariant subspace im $H^{-1} D P_{0} \cdots P_{\mu-1} D^{-} H$ with the ODE obtained by changing the variable $u=H \tilde{u}$ in the inherent regular $O D E$ (3.4) of (4.1) and scaling the resulting $O D E$ by $H^{-1}$.

(iii) For $i \geq 0$, it holds that

$$
\tilde{S}_{i}=S_{i}=\left(F_{i} \cdots F_{0}\right)^{-1} S_{i}, \quad \tilde{S}_{i} \cap \tilde{N}_{i}=\left(F_{i} \cdots F_{0}\right)^{-1}\left(S_{i} \cap N_{i}\right) .
$$

P r o o f : Assertion (i) has already been proved.

To verify Assertion (iii) we derive

$$
\begin{aligned}
& S_{i}=\operatorname{ker} W_{i} B_{i}=\operatorname{ker} W_{i} B_{0} \text { and, with } \tilde{W}_{i}=W_{i} \\
& \tilde{S}_{i}=\operatorname{ker} \tilde{W}_{i} \tilde{B}_{i}=\operatorname{ker} \tilde{W}_{i} \tilde{B}_{0}=\operatorname{ker} W_{i} \tilde{B}_{0}=\operatorname{ker} W_{i} B_{0},
\end{aligned}
$$

hence, $S_{i}=\tilde{S}_{i}$.

Moreover, we have

$$
\begin{aligned}
\left(F_{i} \cdots F_{0}\right)^{-1} S_{i} & =\operatorname{ker} W_{i} B_{i} F_{i} \cdots F_{0}=\operatorname{ker} W_{i} B_{0} P_{0} \cdots P_{i-1} F_{i} \cdots F_{0} \\
& =\operatorname{ker} W_{i} B_{0} P_{0} \cdots P_{i-1}=\operatorname{ker} W_{i} B_{i}=S_{i} .
\end{aligned}
$$

Now, by Lemma 4.2, it holds that $\tilde{N}_{i}=\left(F_{i} \cdots F_{0}\right)^{-1} N_{i}$, and Assertion (iii) is proved. It remains to show (ii).

Compute $\tilde{B} \tilde{D}^{-}=B D^{-} H+A D D^{-} H^{\prime} H^{-1} R H$,

$\tilde{D} \tilde{P}_{0} \cdots \tilde{P}_{\mu-1} \tilde{G}_{\mu}^{-1}=H^{-1} D P_{0} \cdots P_{\mu-1} G_{\mu}^{-1}$,

$\tilde{D} \tilde{P}_{0} \cdots \tilde{P}_{\mu-1} \tilde{G}_{\mu}^{-1} \tilde{B} \tilde{D}^{-}=H^{-1} D P_{0} \cdots P_{\mu-1} G_{\mu}^{-1} B D^{-} H+H^{-1} D P_{0} \cdots P_{\mu-1} D^{-} H^{\prime} H^{-1} R H$. 
Therefore, the inherent regular ODE of (4.7) is

$$
\begin{aligned}
& \tilde{u}^{\prime}-\left(H^{-1} D P_{0} \cdots P_{\mu-1} D^{-} H\right)^{\prime} \tilde{u}+H^{-1} D P_{0} \cdots P_{\mu-1} G_{\mu}^{-1} B D^{-} H \tilde{u} \\
& \quad+H^{-1} D P_{0} \cdots P_{\mu-1} D^{-} H^{\prime} H^{-1} R H \tilde{u}=H^{-1} D P_{0} \cdots P_{\mu-1} G_{\mu}^{-1} q .
\end{aligned}
$$

Rearranging the terms in (4.13) gives

$$
\begin{aligned}
\tilde{u}^{\prime} & -H^{-1 \prime} D P_{0} \cdots P_{\mu-1} D^{-} H \tilde{u}-H^{-1}\left(D P_{0} \cdots P_{\mu-1} D^{-}\right)^{\prime} H \tilde{u} \\
& -H^{-1} D P_{0} \cdots P_{\mu-1} D^{-} H^{\prime}\left(I-H^{-1} R H\right) \tilde{u}+H^{-1} D P_{0} \cdots P_{\mu-1} G_{\mu}^{-1} B D^{-} H \tilde{u} \\
& =H^{-1} D P_{0} \cdots P_{\mu-1} G_{\mu}^{-1} q .
\end{aligned}
$$

Along the basic invariant subspace $i m H^{-1} D P_{0} \cdots P_{\mu-1} D^{-} H$ it holds that

$$
\tilde{u}=H^{-1} D P_{0} \cdots P_{\mu-1} D^{-} H \tilde{u}, \quad\left(I-H^{-1} R H\right) \tilde{u}=0,
$$

such that (4.14) simplifies to

$$
\begin{aligned}
\tilde{u}^{\prime}+H^{-1} H^{\prime} \tilde{u} & -H^{-1}\left(D P_{0} \cdots P_{\mu-1} D^{-}\right)^{\prime} H \tilde{u} \\
& +H^{-1} D P_{0} \cdots P_{\mu-1} G_{\mu}^{-1} B D^{-} H \tilde{u}=H^{-1} D P_{0} \cdots P_{\mu-1} G_{\mu}^{-1} q .
\end{aligned}
$$

The same regular ODE will be obtained by changing the variable $u=H \tilde{u}$ in the inherent regular ODE of (4.1), namely, in (3.4), and premultiplying the resulting equation by $H^{-1}$.

Corollary 4.4 (i) Each DAE that results from a constant coefficient DAE with a regular matrix pencil $\{E, F\}$ via transforming the variables, scaling the equation and refactorizing the leading term is a regular DAE with index $\mu=$ ind $\{E, F\}$.

(ii) Each DAE that results in this way from an $S S C F-D A E$ (2.12) is regular with index $\mu=\operatorname{ind}(N)$.

Applying Corollary 4.4 we know the index of the DAE (1.9) to be $\mu=$ ind $\{E, F\}$.

Now, considering a simple example we are able to demonstrate that the second term in $B_{i}$ has to be given, in fact.

\section{Example 4.1:}

$\overline{\text { We put the DAE }}$

$$
\begin{aligned}
x_{2}^{\prime}+x_{1} & =q_{1} \\
x_{3}^{\prime}+x_{2} & =q_{2}, \\
x_{3} & =q_{3},
\end{aligned}
$$

which obviously has index 3 , in the form (4.1) by taking

$$
A=\left(\begin{array}{ll}
1 & 0 \\
0 & 1 \\
0 & 0
\end{array}\right), \quad D=\left(\begin{array}{lll}
0 & 1 & 0 \\
0 & 0 & 1
\end{array}\right), \quad B=I .
$$

Choose $H(t)=\left(\begin{array}{cc}1 & 0 \\ \eta t & 1\end{array}\right), H(t)^{-1}=\left(\begin{array}{cc}1 & 0 \\ -\eta t & 1\end{array}\right), \quad K(t)=\left(\begin{array}{ccc}1 & 0 & 0 \\ 0 & 1 & 0 \\ 0 & \eta t & 1\end{array}\right)$, refactorize with $H$ and transform $x=K \tilde{x}$. 
Compute

$$
\begin{aligned}
& \tilde{A}=A H=\left(\begin{array}{cc}
1 & 0 \\
\eta t & 1 \\
0 & 0
\end{array}\right), \quad \tilde{D}=H^{-1} D K=\left(\begin{array}{lll}
0 & 1 & 0 \\
0 & 0 & 1
\end{array}\right)=D \\
& \tilde{B}=B K+A H^{\prime} H^{-1} D K=\left(\begin{array}{ccc}
1 & 0 & 0 \\
0 & 1+\eta & 0 \\
0 & \eta t & 1
\end{array}\right)
\end{aligned}
$$

such that the new DAE $\tilde{A}(\tilde{D} \tilde{x})^{\prime}+\tilde{B} \tilde{x}=q$ reads in detail

$$
\begin{aligned}
\tilde{x}_{2}^{\prime}+\tilde{x}_{1} & =q_{1}, \\
\eta t \tilde{x}_{2}^{\prime}+\tilde{x}_{3}^{\prime}+(1+\eta) \tilde{x}_{2} & =q_{2}, \\
\eta t \tilde{x}_{2}+\tilde{x}_{3} & =q_{3} .
\end{aligned}
$$

By our expectation, which is confirmed by Corollary 4.4, this DAE should have index 3 independently of the value of $\eta$. Compute the corresponding sequence $4 \mathrm{pt} 0.8 \mathrm{~mm}$

$$
\begin{aligned}
& \tilde{G}_{0}=\left(\begin{array}{ccc}
0 & 1 & 0 \\
0 & \eta t & 1 \\
0 & 0 & 0
\end{array}\right), \quad \tilde{B}_{0}=\left(\begin{array}{ccc}
1 & 0 & 0 \\
0 & 1+\eta & 0 \\
0 & \eta t & 1
\end{array}\right), \quad \tilde{Q}_{0}=\left(\begin{array}{lll}
1 & & \\
& 0 & \\
& 0
\end{array}\right), \\
& \tilde{G}_{1}=\left(\begin{array}{ccc}
1 & 1 & 0 \\
0 & \eta t & 1 \\
0 & 0 & 0
\end{array}\right), \quad \tilde{Q}_{1}=\left(\begin{array}{ccc}
0 & -1 & 0 \\
0 & 1 & 0 \\
0 & -\eta t & 0
\end{array}\right), \quad \tilde{B}_{0} \tilde{P}_{0} \tilde{Q}_{1}=\left(\begin{array}{ccc}
0 & 0 & 0 \\
0 & 1+\eta & 0 \\
0 & 0 & 0
\end{array}\right), \\
& \tilde{G}_{2}=\left(\begin{array}{ccc}
1 & 1 & 0 \\
0 & 1+\eta t & 1 \\
0 & 0 & 0
\end{array}\right), \quad \tilde{\Gamma}_{2}:=\tilde{G}_{1}+\tilde{B}_{0} \tilde{P}_{0} \tilde{Q}_{1}=\left(\begin{array}{ccc}
1 & 1 & 0 \\
0 & 1+\eta+\eta t & 1 \\
0 & 0 & 0
\end{array}\right) .
\end{aligned}
$$

Recall that $\tilde{G}_{3}$ is nonsingular if and only if the intersection $\tilde{N}_{2} \cap \tilde{S}_{2}$ is trivial (cf. (2.5)). Actually, we have $\tilde{N}_{2}=\left\{z \in \mathbb{R}^{3}: z_{1}+z_{2}=0,(1+\eta t) z_{2}+z_{3}=0\right\}, \tilde{S}_{2}=\left\{z \in \mathbb{R}^{3}\right.$ : $\left.\eta t z_{2}+z_{3}=0\right\}$, thus $\tilde{N}_{2} \cap \tilde{S}_{2}=0$, i.e., system (4.16) is a regular DAE with index $\mu=3$ by our definition, independently of the parameter value $\eta$. On the other hand, if we drop the second term in $\tilde{B}_{1}=\tilde{B}_{0} \tilde{P}_{0}-\tilde{G}_{1} \tilde{D}^{-}\left(\tilde{D} \tilde{P}_{1} \tilde{D}^{-}\right)^{\prime} D$ we obtain $\tilde{\Gamma}_{2}=\tilde{G}_{1}+\tilde{B}_{0} \tilde{P}_{0} \tilde{Q}_{1}$ instead of $\tilde{G}_{2}$. Now, for $\eta=-1$ we have

$\operatorname{ker} \tilde{\Gamma}_{2}=\left\{z \in \mathbb{R}^{3}: z_{1}+z_{2}=0,-t z_{2}+z_{3}=0\right\}, \tilde{S}_{2}=\left\{z \in \mathbb{R}^{3}:-t z_{2}+z_{3}=0\right\}$, which leads to $\operatorname{ker} \tilde{\Gamma}_{2} \cap \tilde{S}_{2}=\operatorname{ker} \tilde{\Gamma}_{2} \neq 0$.

This means that, if we used such a simpler version of $B_{i}$ (as it is used in the constant coefficient case), then the resulting criterion would not recognize the index properly.

Remark 4.5 When checking the index of a given DAE (4.1), sometimes it might be more convenient to consider the rank of the matrix $\Gamma_{i+1}:=G_{i}+B_{i-1} P_{i-1} Q_{i}$ instead of rank $G_{i+1}$ (cf. (2.3)). Instead of testing the nonsingularity of $G_{i+1}$ it might be easier to check whether $N_{i} \cap S_{i}=0$ holds (cf. (2.5)).

Return once more to the above Example 4.1. Now we use the full rank rectangular matrix

$H(t)=\left(\begin{array}{ccc}1 & 0 & 0 \\ \eta t & 1 & 0\end{array}\right)$ and its generalized inverse $H(t)^{-}=\left(\begin{array}{cc}1 & 0 \\ -\eta t & 1 \\ 0 & 0\end{array}\right)$ 
instead of the quadratic nonsingular matrix and its inverse applied before. This leads to

$$
\tilde{A}=A H=\left(\begin{array}{ccc}
1 & 0 & 0 \\
\eta t & 1 & 0 \\
0 & 0 & 0
\end{array}\right), \quad \tilde{D}=H^{-} D K=\left(\begin{array}{ccc}
0 & 1 & 0 \\
0 & 0 & 1 \\
0 & 0 & 0
\end{array}\right)
$$

hence, we have changed the dimension $n=2$ to $\tilde{n}=3$. Observe that $H(t) H(t)^{-}=$ $R=I$ is valid. In detail, the same system (4.16) results, which is a regular index 3 DAE.

Also in general, we may refactorize the leading term of (4.1) not only by means of nonsingular matrix functions. Instead we may also use possibly rectangular matrix functions

$$
H \in C^{1}\left(\mathcal{I}, L\left(\mathbb{R}^{s}, \mathbb{R}^{n}\right), \quad r \leq s \leq m,\right.
$$

which have generalized inverses

$$
H^{-} \in C^{1}\left(\mathcal{I}, L\left(\mathbb{R}^{n}, \mathbb{R}^{s}\right)\right.
$$

such that the additional condition

$$
R H H^{-} R=R
$$

is satisfied. Thereby, $r$ denotes the common constant rank of the matrix functions $A$ and $D$ that are supposed to be well matched. Condition (4.19) immediately implies $A D=A H H^{-} D$. The coefficients of the DAE resulting from this refactorization are

$$
\tilde{A}=A H, \quad \tilde{D}=H^{-} D, \quad \tilde{B}=B+A D D^{-}(R H)^{\prime} H^{-} D .
$$

Due to condition (4.19), the coefficients $\tilde{A}$ and $\tilde{D}$ are well matched, too. It holds that $\tilde{R}=H^{-} R H$. Since $\tilde{A} \tilde{D}=A D$ is given, one can choose $\tilde{P}_{0}=P_{0}$. This yields the relation $\tilde{D}^{-}=D^{-} H$.

Theorem 4.6 Let (4.1) be a regular DAE with index $\mu$.

(i) Then the DAE (4.7) refactorized by (4.17), (4.18) (4.19) is also regular with index $\mu$.

(ii) The resulting regular $O D E$ of the DAE (4.7) has im $H^{-} D P_{0} \cdots P_{\mu-1} D^{-} H$ as the basic invariant subspace. It coincides along this subspace with the ODE obtained by changing the variable $\tilde{u}=H^{-} u$ in the inherent regular ODE (3.4) of (4.1) and then scaling by $\mathrm{H}^{-}$.

\section{Proof:}

(i) Form both sequences of matrix functions and consider how they are related. We have

$$
\begin{aligned}
& \tilde{G}_{0}=\tilde{A} \tilde{D}=A D=G_{0}, \quad \tilde{Q}_{0}=Q_{0} \\
& \tilde{G}_{1}=\tilde{G}_{0}+\tilde{B}_{0} \tilde{Q}_{0}=G_{0}+\tilde{B}_{0} Q_{0}=G_{0}+B Q_{0}=G_{1}, \quad \tilde{Q}_{1}=Q_{1},
\end{aligned}
$$


$\tilde{G}_{2}=G_{2}+G_{0} Q_{1} D^{-}(R H)^{\prime} H^{-} D Q_{1}=G_{2} F_{2}$

with the nonsingular factor

$$
F_{2}=I+P_{1} P_{0} Q_{1} D^{-}(R H)^{\prime} H^{-} D Q_{1}=I-Q_{0} Q_{1} D^{-}(R H)^{\prime} H^{-} D Q_{1} .
$$

Letting $F_{0}=I, \quad F_{1}=I$, the relation $\tilde{G}_{i}=G_{i} F_{i} \cdots F_{0}$ is now valid for $i \leq 2$. It can be proved for $i \geq 2$ in the same way as Lemma 4.2 is proved if the expression $D^{-} H^{\prime} H^{-1} D$ is now replaced by $D^{-}(R H)^{\prime} H^{-} D$ and if it is taken into account that, due to condition (4.19), $D^{-} H\left(H^{-} R\right)^{\prime} D=D^{-} R H\left(H^{-} R\right)^{\prime} D=D^{-}\left(R H H^{-} R\right)^{\prime} D-$ $D^{-}(R H)^{\prime} H^{-} D=D^{-} R^{\prime} D-D^{-}(R H)^{\prime} H^{-} D=-D(R H)^{\prime} H^{-} D$ holds true.

(ii) In the same way as relation (4.12) is verified, we obtain now $\tilde{P}_{0} \cdots \tilde{P}_{i}=P_{0} \cdots P_{i}$ and thus $\tilde{D} \tilde{P}_{0} \cdots \tilde{P}_{i} \tilde{D}^{-}=H^{-} D P_{0} \cdots P_{i} D^{-} H, i \geq 0$. In particular, for $i=\mu-1$, the basic invariant subspace is described as claimed above.

The inherent regular ODE of the refactorized DAE is

$$
\begin{aligned}
\tilde{u}^{\prime}- & \left(H^{-} D P_{0} \cdots P_{\mu-1} D^{-} H\right)^{\prime} \tilde{u}+H^{-} D P_{0} \cdots P_{\mu-1} G_{\mu}^{-1} B D^{-} H \tilde{u} \\
& +H^{-} D P_{0} \cdots P_{\mu-1} D^{-}(R H)^{\prime} H^{-} R H \tilde{u}=H^{-} D P_{0} \cdots P_{\mu-1} G_{\mu}^{-1} q .
\end{aligned}
$$

Along the basic invariant subspace, we make use of the properties $\tilde{u}=H^{-} R H \tilde{u}=$ $\tilde{R} \tilde{u}=\tilde{u}, \quad \tilde{u}=H^{-} D P_{0} \cdots P_{\mu-1} D^{-} H \tilde{u}$ such that (4.21) implies

$$
\begin{aligned}
\tilde{u}^{\prime}-\left(H^{-} R\right)^{\prime} R H \tilde{u} & -H^{-} R\left(D P_{0} \cdots P_{\mu-1} D^{-}\right)^{\prime} R H \tilde{u} \\
& +H^{-} D P_{0} \cdots P_{\mu-1} G_{\mu}^{-1} B D^{-} H \tilde{u}=H^{-} D P_{0} \cdots P_{\mu-1} G_{\mu}^{-1} q .
\end{aligned}
$$

The same ODE would be obtained by considering the inherent regular ODE of (4.1)

$$
u^{\prime}-\left(D P_{0} \cdots P_{\mu-1} D^{-}\right)^{\prime} u+D P_{0} \cdots P_{\mu-1} G_{\mu}^{-1} B D^{-} u=D P_{0} \cdots P_{\mu-1} G_{\mu}^{-1} q
$$

on its basic invariant subspace, i.e., with $u=D P_{0} \cdots P_{\mu-1} D^{-} u, u=R u$, which leads to $u^{\prime}-R^{\prime} u-R\left(D P_{0} \cdots P_{\mu-1} D^{-}\right)^{\prime} u+D P_{0} \cdots P_{\mu-1} G_{\mu}^{-1} B D^{-} u=D P_{0} \cdots P_{\mu-1} G_{\mu}^{-1} q$, by transforming $\tilde{u}:=H^{-} u$ and then premultiplying by $H^{-}$. Thereby, we take advantage of the relations $R H \tilde{u}=R H H^{-} u=R H H^{-} R u=u, H^{-} R H \tilde{u}=H^{-} u=\tilde{u}$.

Remark 4.7 In particular, Theorem 4.6 enables to turn from rank-deficient coefficients $A$ and $D$ (with $n>r$ ) to full-rank versions $\tilde{A}$ and $\tilde{D}$ by choosing $\tilde{n}=s=r$. Then, because of $\operatorname{ker} \tilde{A}=0$, im $\tilde{D}=\mathbb{R}^{r}$, it holds that $\tilde{R}=I$. Note that this is preferable in view of numerical integration methods (cf. [HiMäTi], [Mä]).

\section{$5 \quad$ Further discussion and conclusions}

With this paper we are aiming at an understanding of regularity for DAEs (1.1) with only continuous coefficients $A, D$ and $B$ that takes its origin in classical ODE theory and at the formulation of index criteria in terms of these coefficients. The proposed notion of regularity with index $\mu$ generalizes the Kronecker index, the global index (cf. [GePe] and Remark 2.8) as well as the tractability index (cf. Remark 2.6). 
In comparison to the latter one it has to be emphasized that $A$ and $D$ are now allowed to be arbitrary, continuous, possibly rectangular well-matched matrix functions, whereas, in the case of the tractability index (e.g. [Mä]) $D$ has to be a quadratic, continuously differentiable projector function.

Recent investigations ([ReMaBa]) on the so-called structural index have shown that the related, well known Pantelides algorithm can provide, for linear constant coefficient DAEs already, a structural index that might be either far less than the Kronecker index or even much larger. Not even index one is identified precisely. Hence, relative simple, practicable and at the same time reliable index criteria are all the more important. For circuit simulation, an index-monitor basing on the tractability index has proved its value ([Es et all]). Regularity with index $\mu$ holds similar powers and seems to be even simpler in handling.

When orienting on smooth (non-analytical) coefficients and starting from $C^{1}$-solutions, comparisons with concepts for standard form DAEs with respect to the algebraic conditions (e.g. rank conditions) can be made via

$$
A D x^{\prime}+\left(B+A D^{\prime}\right) x=q .
$$

Due to [RaRh, Theorem 7.1] regularity with index 1 coincides, for (1.1), with the complete reducibility with index 1 (algebraically). Even the further constant-rank conditions from [RaRh] seem to coincide with those of Definition 2.4. A detailed investigation is still to come.

Concerning the strangeness index concept ([KuMe]) let us point out that, by our understanding of regularity, just undetermined parts shall be excluded and DAEs like those of Example 2.2 will be characterized as not being regular exclusively. In contrast, in $[\mathrm{KuMe}]$ undetermined parts are considered in more detail and Example 2.2 has the strangeness index 1 and the further characteristic quantity $u_{1}=1$. Moreover, the relations $r_{0}^{K M}=r_{0}$ and $a_{0}:=\operatorname{rank}\left(Z^{*} B T\right)=r_{1}-r_{0}$ (the columns of $T$ and $Z$ span $\operatorname{ker}(A D)$ and $i m(A D)^{\perp}$, respectively) hold true for the quantities $r_{0}^{K M}, a_{0}$ used in [KuMe] for (5.1). Hence, $r_{0}$ and $r_{1}$ are constant on the given interval if $r_{0}^{K M}$ and $a_{0}$ are so. The further constant rank conditions used in [KuMe] seem to coincide with those from Definition 2.4, too, however, this requires a deeper investigation. By all known odds, except for smoothness conditions a regular index $\mu$ DAE (1.1) seems to yield a standard form DAE (5.1) which has strangeness index $\nu=\mu-1$ and the characteristic quantities $r_{\nu}^{K M}=d_{\nu}^{K M}=d_{\mu}\left(\right.$ cf. Remark 3.2), $a_{\nu}=m=d_{\mu}, u_{\nu}=0$.

For instance in $[\mathrm{Ca}],[\mathrm{CaPe}]$ great store is set by working without any rank conditions for $A D=G_{0}$. Canonical standard forms are a reasonable example of this. As already mentioned in Section 2, we want to consider all points in which rank changes occur to be singularities (perhaps of harmless character). This is consistent with the point of view expressed in [RaRh] and [KuMe]. A detailed characterization of singularities is still to come.

No doubt, in case the constant rank conditions are perturbed, the corresponding procedures can be realized on constant rank subintervals first and then the subresults can be combined. The solution representations resulting from regularity with index $\mu$, like 
(3.2), (3.3), seem to be well suited for further investigations in this direction.

Example 5.1: Consider the DAE

$$
\left(\begin{array}{cc}
0 & \alpha \\
\beta & 0
\end{array}\right) x^{\prime}+x=q
$$

with continuous real functions $\alpha, \beta$ such that $\alpha(t) \beta(t)=0, t \in \mathcal{I}$.

Denote by $\chi_{f}$ the characteristic function corresponding to a given real function $f$, i.e., for $t \in \mathcal{I}, \chi_{f}(t)=1$ if $f(t) \neq 0, \chi_{f}(t)=0$ if $f(t)=0$.

On each subinterval where $\left(\begin{array}{cc}0 & \alpha(t) \\ \beta(t) & 0\end{array}\right)$ is of constant rank we rewrite the standard form DAE (5.2) with a properly stated leading term by means of

$$
A=\left(\begin{array}{cc}
0 & \alpha \\
\beta & 0
\end{array}\right), \quad D=\left(\begin{array}{cc}
\chi_{\alpha} & 0 \\
0 & \chi_{\beta}
\end{array}\right), B=I .
$$

Compute, on each subinterval, $D=R=P_{0}=D^{-}$, further

$$
\begin{aligned}
& G_{0}=\left(\begin{array}{cc}
0 & \alpha \\
\beta & 0
\end{array}\right), Q_{0}=\left(\begin{array}{cc}
1-\chi_{\beta} & 0 \\
0 & 1-\chi_{\alpha}
\end{array}\right), G_{1}=\left(\begin{array}{cc}
1-\chi_{\beta} & \alpha \\
\beta & 1-\chi_{\alpha}
\end{array}\right), \\
& Q_{1}=\left(\begin{array}{cc}
\chi_{\beta} & -\alpha \\
-\beta & \chi_{\alpha}
\end{array}\right), G_{2}=\left(\begin{array}{cc}
1 & \alpha \\
\beta & 1
\end{array}\right) \\
& D P_{1} D^{-}=0, \quad \operatorname{det} G_{2}=1,\left(Q_{0} P_{1}+P_{0} Q_{1}\right) G_{2}^{-1}=I \\
& D Q_{1} G_{2}^{-1}=\left(\begin{array}{cc}
\chi_{\beta} & 0 \\
0 & \chi_{\alpha}
\end{array}\right), Q_{0} Q_{1} D^{-}=\left(\begin{array}{cc}
0 & -\alpha \\
-\beta & 0
\end{array}\right) .
\end{aligned}
$$

Observe that, for trivial reasons, $D P_{1} D^{-}$has a smooth extension on the entire interval $\mathcal{I}$.

On subintervals where both $\alpha$ and $\beta$ vanish, it holds that $G_{1}=I$ and we have regularity with index 1. On subintervals where one of these functions vanishes but the other one does not so, we have regularity with index 2 . In all cases we can use the nonsingularity of $G_{2}$ and apply the solution expression (3.2) on each subinterval. This yields (since $\left.D P_{1} D^{-}=0\right)$

$$
x=\left(P_{0} Q_{1}+Q_{0} P_{1}\right) G_{2}^{-1} q+Q_{0} Q_{1} D^{-}\left(D Q_{1} G_{2}^{-1} q\right)
$$

thus

$$
x=q-\left(\begin{array}{cc}
\alpha & \left(\chi_{\alpha} q_{2}\right)^{\prime} \\
\beta & \left(\chi_{\beta} q_{2}\right)^{\prime}
\end{array}\right) .
$$

Hence, for each $q$ that is continuous on the the entire interval and has $C^{1}$-components $q_{2}$ resp. $q_{1}$ on the corresponding subintervals, this DAE is uniquely solvable. However, the solution $x$ may undergo discontinuities at the exceptionary points where the rank conditions fail. For example, if $\mathcal{I}=[-1,1], \beta(t)=0$ for $t \in \mathcal{I}, \alpha(t)=0$ for $t \in[-1,0], \quad \alpha(t)=t^{\frac{1}{3}}$ for $t>0$, further $q_{1}(t)=0$ for $t \in[-1,1], \quad q_{2}(t)=0$ for $t \in[-1,0], \quad q_{2}(t)=t^{\frac{1}{3}}$, there is a continuous extension of $x_{2}(t)=q_{2}(t)$ on $\mathcal{I}$. However, the first component is $x_{1}(t)=0$ on $[-1,0)$ and $x_{1}(t)=-\frac{1}{3} t^{-\frac{1}{3}}$ on $(0,1]$ so that there is no solution that is continuous on $\mathcal{I}$. 
If there is more smoothness within the coefficients, e.g. $\alpha, \beta \in C^{1}(\mathcal{I}, \mathbb{R}), \quad q \in$ $C^{1}\left(\mathcal{I}, \mathbb{R}^{2}\right)$, then, on the subintervals, (5.3) may be rewritten as $x=q-\left(\begin{array}{ll}\alpha & q_{2}^{\prime} \\ \beta & q_{1}^{\prime}\end{array}\right)$, which posesses a continuous extension on $\mathcal{I}$ (cf. [BrCaPe, Example 2.4.3]).

In spite of the possible discontinuities in the solution we consider the singularities in Example 5.1. to be harmless in a way.

A further problem that has not been sufficiently solved even for regular DAEs is that of continuous solution components for inhomogeneities $q$ with jumps. This plays an essential role in sensitivity analysis and for optimal control problems. Here, too, we are in need of sharp solvability statements in case of low smoothness.

\section{Appendix}

\subsection{Proof of Proposition 2.10}

For a DAE (2.1) with properly stated leading term, and for a fixed $i_{*}>0$, we consider the continuous matrix functions $G_{0}, G_{1}, \ldots, G_{i_{*}}$ determined by (2.2) such that $Q_{i} Q_{j}=0, \quad 0 \leq j \leq i \leq i_{*}-1$ is satisfied and it holds that $\left(N_{0} \oplus \cdots \oplus N_{i_{*}-1}\right) \cap N_{i_{*}}=0$. The projector functions $Q_{0}, \ldots, Q_{i_{*}-1}$ are assumed to be continuous but the $D P_{0} \ldots P_{i} D^{-}$, $i=0, \ldots, i_{*}-1$, to be continuously differentiable.

Now we suppose that there are two different continuous projector functions $Q_{i_{*}}$ and $\tilde{Q}_{i_{*}}$ onto $N_{i_{*}}=\operatorname{ker} G_{i_{*}}$ such that $Q_{i_{*}} Q_{j}=\tilde{Q}_{i_{*}} Q_{j}=0$ is valid for $j=0, \ldots, i_{*}-1$, and both $D P_{0} \cdots P_{i_{*}} D^{-}$and $D P_{0} \cdots P_{i_{*}-1} \tilde{P}_{i_{*}} D^{-}$are continuously differentiable. We are going to compare the resulting two matrix function sequences.

Taking into account the simple basic relations

$$
Q_{i_{*}} \tilde{Q}_{i_{*}}=\tilde{Q}_{i_{*}}, \quad P_{i_{*}} \tilde{P}_{i_{*}}=P_{i_{*}}, \quad \tilde{Q}_{i_{*}} Q_{i_{*}}=Q_{i_{*}}, \quad \tilde{P}_{i_{*}} P_{i_{*}}=\tilde{P}_{i_{*}}
$$

we find the following further properties to be used later on frequently:

$$
\begin{aligned}
& P_{0} \cdots P_{i_{*}-1} \tilde{P}_{i_{*}}=P_{0} \cdots P_{i_{*}-1} P_{i_{*}}+P_{0} \cdots P_{i_{*}-1} Q_{i_{*}} \tilde{P}_{i_{*}}, \\
& \begin{aligned}
Q_{i_{*}} \tilde{P}_{i_{*}} & =Q_{i_{*}} \tilde{P}_{i_{*}} P_{i_{*}}=\left(Q_{i_{*}}-Q_{i_{*}} \tilde{Q}_{i_{*}}\right) P_{i_{*}} \\
& =\left(Q_{i_{*}} P_{0} \cdots P_{i_{*}-1}-Q_{i_{*}} \tilde{Q}_{i_{*}} P_{0} \cdots P_{i_{*}-1}\right) P_{i_{*}} \\
& =\left(Q_{i_{*}}-Q_{i_{*}} \tilde{Q}_{i_{*}}\right) P_{0} \cdots P_{i_{*}-1} P_{i_{*}}=Q_{i_{*}} \tilde{P}_{i_{*}} P_{0} \cdots P_{i_{*}},
\end{aligned} \\
& Q_{i_{*}} \tilde{P}_{i_{*}} Q_{j}=0 \quad \text { for } j=0, \ldots, i_{*}-1, \\
& Q_{i_{*}} \tilde{P}_{i_{*}}=-Q_{i_{*}} \tilde{Q}_{i_{*}} P_{0} \cdots P_{i_{*}-1} P_{i_{*}}=-\tilde{Q}_{i_{*}} P_{i_{*}} .
\end{aligned}
$$

From (6.1) we know $D P_{0} \cdots P_{i_{*}-1} Q_{i_{*}} \tilde{P}_{i_{*}} D^{-}$to be continuously differentiable, too.

The consecutive matrix functions (cf. (2.2)) $G_{i_{*}+1}=G_{i_{*}}+B_{i_{*}} Q_{i_{*}}$ and $\tilde{G}_{i_{*}+1}=G_{i_{*}}+$ $\tilde{B}_{i_{*}} \tilde{Q}_{i_{*}}$ satisfy the relation

$$
\tilde{G}_{i_{*}+1}=G_{i_{*}+1} M_{i_{*}+1}
$$


with the factor

$M_{i_{*}+1}=P_{i_{*}}+\tilde{Q}_{i_{*}}+P_{i_{*}} P_{0} \cdots P_{i_{*}-1} Q_{i_{*}} \tilde{P}_{i_{*}} D^{-}\left(D P_{0} \cdots P_{i_{*}-1} Q_{i_{*}} D^{-}\right)^{\prime} D P_{0} \cdots P_{i_{*}-1} Q_{i_{*}} \tilde{Q}_{i_{*}}$.

Because of $P_{i_{*}}+\tilde{Q}_{i_{*}}=I+\tilde{Q}_{i_{*}} P_{i_{*}}$ and

$P_{i_{*}} P_{0} \cdots P_{i_{*}-1} Q_{i_{*}}=P_{0} \cdots P_{i_{*}-1} Q_{i_{*}}-Q_{i_{*}}=\left(I-Q_{0}\right) \cdots\left(I-Q_{i_{*}-1}\right) Q_{i_{*}}-Q_{i_{*}}=: \sum_{l=0}^{i_{*}-1} Q_{l} \mathcal{C}_{l} Q_{i_{*}}$ we may rewrite $M_{i_{*}+1}$ as

$$
M_{i_{*}+1}=I+\tilde{Q}_{i_{*}} P_{i_{*}}+\sum_{l=0}^{i_{*}-1} Q_{l} \mathfrak{B}_{i_{*}+1, l} \tilde{Q}_{i_{*}}
$$

with continuous terms

$$
\mathfrak{B}_{i_{*}+1, l}=\mathcal{C}_{l} Q_{i_{*}} \tilde{P}_{i_{*}} D^{-}\left(D P_{0} \cdots P_{i_{*}-1} Q_{i_{*}} D^{-}\right)^{\prime} D P_{0} \cdots P_{i_{*}-1} Q_{i_{*}}
$$

The matrix function $M_{i_{*}+1}$ remains nonsingular,

$$
M_{i_{*}+1}^{-1}=I=\tilde{Q}_{i_{*}} P_{i_{*}}-\sum_{l=0}^{i_{*}-1} Q_{l} \mathfrak{B}_{i_{*}+1, l} Q_{i_{*}} .
$$

In the consequence $G_{i_{*}+1}$ and $\tilde{G}_{i_{*}+1}$ have the same rank everywhere. If both have constant rank $r_{i_{*}+1}$, and if the first sequence may be continued by a continuous projector function $Q_{i_{*}+1}$ onto $N_{i_{*}+1}=\operatorname{ker} G_{i_{*}+1}$ such that $D P_{0} \cdots P_{i_{*}} D^{-}$is continuously differentiable and $Q_{i_{*}+1} Q_{l}=0$ is fulfilled for $l=0, \ldots, i_{*}$, then, letting $\tilde{Q}_{i_{*}+1}=M_{i_{*}+1}^{-1} Q_{i_{*}+1} M_{i_{*}}$, the second sequence may be continued, too, at the same time. Namely, we have $\tilde{Q}_{i_{*}+1}=M_{i_{*}+1}^{-1} Q_{i_{*}+1}$, thus $\tilde{Q}_{i_{*}+1} Q_{j}=0$ for $j=0, \ldots, i_{*}$, and $\tilde{Q}_{i_{*}+1} \tilde{Q}_{i_{*}}=\tilde{Q}_{i_{*}+1} Q_{i_{*}} \tilde{Q}_{i_{*}}=0$, further

$$
\begin{aligned}
D P_{0} \cdots \tilde{P}_{i_{*}} \tilde{P}_{i_{*}+1} D^{-} & =D P_{0} \cdots P_{i_{*}+1} D^{-}+D^{-} P_{0} \cdots P_{i_{*}-1} Q_{i_{*}} \tilde{P}_{i_{*}} D^{-} P_{0} \cdots P_{i_{*}+1} D^{-} \\
& =\left(I+D^{-} P_{0} \cdots P_{i_{*}-1} Q_{i_{*}} \tilde{P}_{i_{*}} D^{-}\right)\left(D P_{0} \cdots P_{i_{*}+1} D^{-}\right)
\end{aligned}
$$

which shows the smoothness as well as relation (2.7) at this stage to be transferred from the first sequence to the second one.

Below we shall verify the relation

$$
\tilde{G}_{j}=G_{j} M_{j} \cdots M_{i_{*}+2} M_{i_{*}+1}, \quad j \geq i_{*}+2
$$

with continuous nonsingular matrix functions

$$
\begin{aligned}
M_{k} & =I+Q_{i_{*}} \tilde{P}_{i_{*}} P_{i_{*}+1} \cdots P_{k-2} Q_{k-1}+\sum_{l=0}^{k-2} Q_{l} \mathfrak{B}_{k, l} Q_{k-1} \\
& =\left(I+Q_{i_{*}} \tilde{P}_{i_{*}} P_{i_{*}+1} \cdots P_{k-2} Q_{k-1}\right)\left(I+\sum_{l=0}^{k-2} Q_{l} \mathfrak{B}_{k, l} Q_{k-1}\right), \\
M_{k}^{-1} & =I-Q_{i_{*}} \tilde{P}_{i_{*}} P_{i_{*}+1} \cdots P_{k-2} Q_{k-1}-\sum_{l=0}^{k-1} Q_{l} \mathfrak{B}_{k, l} Q_{k-1},
\end{aligned}
$$

and projector functions

$$
\tilde{Q}_{k}=\left(M_{k} \cdots M_{i_{*}+1}\right)^{-1} Q_{k} M_{k} \cdots M_{i_{*}+1}, \quad k=i_{*}+2, \ldots, j-1 .
$$


Thereby, due to the special structure of $M_{k}$, it holds that $Q_{k} M_{k} \cdots M_{i_{*}+1}=Q_{k}$ as well as

$$
D P_{0} \cdots \tilde{P}_{i_{*}} \tilde{P}_{i_{*}+1} \cdots \tilde{P}_{k} D^{-}=\left(I+D P_{0} \cdots P_{i_{*}-1} Q_{i_{*}} \tilde{P}_{i_{*}} D^{-}\right) D P_{0} \cdots P_{k} D^{-}
$$

$k=i_{*}+2, \ldots, j-1$. Consequently, at each stage $j$ we may repeat the above arguments and we are done.

Now it remains to prove relation (6.7) in fact.

Basically, we have to check this for $j=i_{*}+2$, i.e., $\tilde{G}_{i_{*}+2} M_{i_{*}+1}^{-1}=G_{i_{*}+2} M_{i_{*}+2}$ with

$$
M_{i_{*}+2}=I+Q_{i_{*}} \tilde{P}_{i_{*}} Q_{i_{*}+1}+\sum_{l=0}^{i_{*}} Q_{l} \mathfrak{B}_{i_{*}+2, l} Q_{i_{*}+1} .
$$

Compute (cf. (2.2))

$$
\begin{aligned}
\tilde{G}_{i_{*}+2} M_{i_{*}+1}^{-1}= & G_{i_{*}+1}+\tilde{B}_{i_{*}+1} M_{i_{*}+1}^{-1} Q_{i_{*}+1} \\
= & G_{i_{*}+1}+\left\{B_{i_{*}-1} P_{i_{*}-1} \tilde{P}_{i_{*}}-G_{i_{*}} D^{-}\left(D P_{0} \cdots P_{i_{*}-1} \tilde{P}_{i_{*}} D^{-}\right)^{\prime} D P_{0} \cdots P_{i_{*}-1} \tilde{P}_{i_{*}}\right. \\
& \left.\quad-\tilde{G}_{i_{*}+1} D^{-}\left(D P_{0} \cdots P_{i_{*}-1} \tilde{P}_{i_{*}} \tilde{P}_{i_{*}+1} D^{-}\right)^{\prime} D P_{0} \cdots P_{i_{*}-1} \tilde{P}_{i_{*}}\right\} M_{i_{*}+1}^{-1} Q_{i_{*}+1} \\
= & G_{i_{*}+1}+B_{i_{*}-1} P_{i_{*}-1} P_{i_{*}} Q_{i_{*}+1}+B_{i_{*}-1} P_{i_{*}-1} Q_{i_{*}} \tilde{P}_{i_{*}} Q_{i_{*}+1} \\
& \quad-G_{i_{*}} D^{-}\left(D P_{0} \cdots P_{i_{*}-1} P_{i_{*}} D^{-}+D P_{0} \cdots P_{i_{*}-1} Q_{i_{*}} \tilde{P}_{i_{*}} D^{-}\right)^{\prime} D P_{0} \cdots P_{i_{*}-1} \tilde{P}_{i_{*}} Q_{i_{*}+1} \\
& \quad-G_{i_{*}+1} D^{-}\left(D P_{0} \cdots P_{i_{*}-1} P_{i_{*}} P_{i_{*}+1} D^{-}+D P_{0} \cdots P_{i_{*}-1} Q_{i_{*}} \tilde{P}_{i_{*}} P_{i_{*}+1} D^{-}\right)^{\prime} \cdot \\
& \quad \cdot D P_{0} \cdots P_{i_{*}-1} \tilde{P}_{i_{*}} Q_{i_{*}+1} \\
& \quad-G_{i_{*}+1}\left(M_{i_{*}+1}-I\right) D^{-}\left(D P_{0} \cdots P_{i_{*}-1} \tilde{P}_{i_{*}} P_{i_{*}+1} D^{-}\right)^{\prime} D P_{0} \cdots P_{i_{*}-1} \tilde{P}_{i_{*}} Q_{i_{*}+1} \\
= & G_{i_{*}+2}\left(I+Q_{i_{*}} \tilde{P}_{i_{*}} Q_{i_{*}+1}\right)-G_{i_{*}+1}\left(M_{i_{*}+1}-I\right) \square Q_{i_{*}+1}+\mathfrak{D}
\end{aligned}
$$

where

$$
\begin{aligned}
\mathfrak{D}= & -G_{i_{*}} D^{-}\left(D P_{0} \cdots P_{i_{*}+1} D^{-}\right)^{\prime} D P_{0} \cdots P_{i_{*}-1} Q_{i_{*}} \tilde{P}_{i_{*}} \tilde{Q}_{i_{*}+1} \\
& -G_{i_{*}} D^{-}\left(D P_{0} \cdots P_{i_{*}-1} Q_{i_{*}} \tilde{P}_{i_{*}} D^{-}\right)^{\prime} D P_{0} \cdots P_{i_{*}-1} \tilde{P}_{i_{*}} \tilde{Q}_{i_{*}+1} \\
& -G_{i_{*}+1} D^{-}\left(D P_{0} \cdots P_{i_{*}-1} Q_{i_{*}} \tilde{P}_{i_{*}} P_{i_{*}+1} D^{-}\right)^{\prime} D P_{0} \cdots P_{i_{*}-1} \tilde{P}_{i_{*}} Q_{i_{*}+1}
\end{aligned}
$$

By construction, we have

$$
\begin{aligned}
G_{i_{*}+1}( & \left.M_{i_{*}+1}-I\right) \square Q_{i_{*}+1}=G_{i_{*}+2} P_{i_{*}+1}\left(\tilde{Q}_{i_{*}} P_{i_{*}}\right. \\
& \left.+\sum_{l=0}^{i_{*}-1} Q_{l} \mathfrak{B}_{i_{*}+1, l} \tilde{Q}_{i_{*}}\right) \square Q_{i_{*}+1}=G_{i_{*}+2} \sum_{l=0}^{i_{*}} Q_{l} \mathfrak{B}_{i_{*}+2, l}^{(1)} Q_{i_{*}+1}
\end{aligned}
$$

Next we try to realize an expression $\mathfrak{D}=\sum_{l=0}^{i_{*}} Q_{l} \mathfrak{B}_{i_{*}+2, l}^{(2)} Q_{i_{*}+1}$ by calculating

$$
\begin{aligned}
\mathfrak{D}= & G_{i_{*}} D^{-} D P_{0} \cdots P_{i_{*}+1} D^{-}\left(D P_{0} \cdots P_{i_{*}-1} Q_{i_{*}} \tilde{P}_{i_{*}} D^{-}\right)^{\prime} D P_{0} \cdots P_{i_{*}} Q_{i_{*}+1} \\
& -G_{i_{*}} D^{-}\left(D P_{0} \cdots P_{i_{*}-1} Q_{i_{*}} \tilde{P}_{i_{*}} D^{-}\right)^{\prime} D P_{0} \cdots P_{i_{*}} Q_{i_{*}+1} \\
& -G_{i_{*}} D^{-}\left(D P_{0} \cdots P_{i_{*}-1} Q_{i_{*}} \tilde{P}_{i_{*}} D^{-}\right)^{\prime} D P_{0} \cdots P_{i_{*}-1} Q_{i_{*}} \tilde{P}_{i_{*}} Q_{i_{*}+1} \\
& +G_{i_{*}+1} D^{-} D P_{0} \cdots P_{i_{*}-1} Q_{i_{*}} \tilde{P}_{i_{*}} P_{i_{*}+1} D^{-}\left(D P_{0} \cdots P_{i_{*}-1} \tilde{P}_{i_{*}} Q_{i_{*}+1} D^{-}\right)^{\prime} D P_{0} \cdots P_{i_{*}} Q_{i_{*}+1}
\end{aligned}
$$


Because of $P_{i_{*}+1} P_{0} \cdots P_{i_{*}-1} Q_{i_{*}}=P_{0} \cdots P_{i_{*}-1} Q_{i_{*}}=\left(I-Q_{0}\right) \cdots\left(I-Q_{i_{*}-1}\right) Q_{i_{*}}$, the last term of $\mathfrak{D}$ has already the form we need.

The first and second terms of $\mathfrak{D}$ give together

$G_{i_{*}}\left\{P_{0} \cdots P_{i_{*}+1}-I\right\} D^{-}\left(D P_{0} \cdots P_{i_{*}-1} Q_{i_{*}} \tilde{P}_{i_{*}} D^{-}\right)^{\prime} D P_{0} \cdots P_{i_{*}} Q_{i_{*}+1}$.

Taking into account that $G_{i_{*}}\left(P_{0} \cdots P_{i_{*}+1}-I\right)=G_{i_{*}+2} P_{i_{*}+1} P_{i_{*}}\left(P_{0} \cdots P_{i_{*}+1}-I\right)=$ $=G_{i_{*}+2}\left(P_{0} \cdots P_{i_{*}+1}-I+Q_{i_{*}+1}+Q_{i_{*}}\right)=G_{i_{*}+2}\left(P_{0} \cdots P_{i_{*}}-I\right) P_{i_{*}+1}+G_{i_{*}+2} Q_{i_{*}}$

we know the term (6.11) to have the right form. The remaining third term of $\mathfrak{D}$ is

$$
\begin{aligned}
- & G_{i_{*}} D^{-}\left(D P_{0} \cdots P_{i_{*}-1} Q_{i_{*}} \tilde{P}_{i_{*}} D^{-}\right)^{\prime} D P_{0} \cdots P_{i_{*}-1} Q_{i_{*}} \tilde{P}_{i_{*}} Q_{i_{*}+1} \\
= & -G_{i_{*}} D^{-}\left(D P_{0} \cdots P_{i_{*}-1} Q_{i_{*}} \tilde{P}_{i_{*}} D^{-}\right)^{\prime} D P_{0} \cdots P_{i_{*}-1} Q_{i_{*}} \tilde{P}_{i_{*}} D^{-} D P_{0} \cdots P_{i_{*}} Q_{i_{*}+1} \\
= & -G_{i_{*}} D^{-}\left(D P_{0} \cdots P_{i_{*}-1} Q_{i_{*}} \tilde{P}_{i_{*}} D^{-} D P_{0} \cdots P_{i_{*}-1} Q_{i_{*}} \tilde{P}_{i_{*}} D^{-}\right)^{\prime} D P_{0} \cdots P_{i_{*}} Q_{i_{*}+1} \\
& +G_{i_{*}} D^{-} D P_{0} \cdots P_{i_{*}-1} Q_{i_{*}} \tilde{P}_{i_{*}} D^{-}\left(D P_{0} \cdots P_{i_{*}-1} Q_{i_{*}} \tilde{P}_{i_{*}} D^{-}\right)^{\prime} D P_{0} \cdots P_{i_{*}} Q_{i_{*}+1} \\
= & G_{i_{*}} P_{0} \cdots P_{i_{*}-1} Q_{i_{*}} \tilde{P}_{i_{*}} D^{-}\left(D P_{0} \cdots P_{i_{*}-1} Q_{i_{*}} \tilde{P}_{i_{*}} D^{-}\right)^{\prime} D P_{0} \cdots P_{i_{*}} Q_{i_{*}+1} .
\end{aligned}
$$

Due to $G_{i_{*}} P_{0} \cdots P_{i_{*}-1} Q_{i_{*}}=G_{i_{*}+2} P_{i_{*}+1} P_{i_{*}} P_{0} \cdots P_{i_{*}-1} Q_{i_{*}}=G_{i_{*}+2}\left(P_{0} \cdots P_{i_{*}-1} Q_{i_{*}}-Q_{i_{*}}\right)$ the third term of $\mathfrak{D}$ has the right form, too, and (6.10) is valid in fact.

Finally, let (6.7) be true for the stages $j=i_{*}+2, \ldots, i$. We have to check the stage $i+1$ by realizing an appropriate $M_{i+1}$ such that

$$
\tilde{G}_{i+1}\left(M_{i} \cdots M_{i_{*}+1}\right)^{-1}=G_{i+1} M_{i+1} .
$$

Notice that from (2.2) it follows that

$$
B_{i}=B_{0} P_{0} \cdots P_{i-1}-\sum_{j=1}^{i} G_{j} D^{-}\left(D P_{0} \cdots P_{j} D^{-}\right)^{\prime} D P_{0} \cdots P_{i-1} .
$$

Derive $P_{0} \cdots P_{i}\left(M_{i} \cdots M_{i_{*}+1}\right)^{-1}=P_{0} \cdots P_{i}$ and $P_{0} \cdots P_{i_{*}-1} \tilde{P}_{i_{*}} \cdots \tilde{P}_{i}=P_{0} \cdots P_{i_{*}-1} \tilde{P}_{i_{*}} P_{i_{*}+1} \cdots P_{i}$ and thus

$$
\begin{gathered}
\tilde{G}_{i+1}\left(M_{i} \cdots M_{i_{*}+1}\right)^{-1}=G_{i}+\tilde{B}_{i}\left(M_{i} \cdots M_{i_{*}+1}\right)^{-1} Q_{i} \\
=G_{i}+B_{0} P_{0} \cdots P_{i-1} Q_{i}+B_{0} P_{0} \cdots P_{i_{*}-1} Q_{i_{*}} \tilde{P}_{i_{*}} P_{i_{*}+1} \cdots P_{i-1} Q_{i} \\
-\sum_{j=1}^{i} G_{j} D^{-}\left(D P_{0} \cdots P_{j} D^{-}\right)^{\prime}\left(D P_{0} \cdots P_{i-1} Q_{i}+D P_{0} \cdots P_{i_{*}-1} Q_{i_{*}} \tilde{P}_{i_{*}} P_{i_{*}+1} \cdots P_{i-1} Q_{i}\right) \\
-\sum_{j=i_{*}}^{i}\left\{\tilde{G}_{j} D^{-}\left(D P_{0} \cdots \tilde{P}_{i_{*}} \cdots P_{j} D^{-}\right)^{\prime}-G_{j} D^{-}\left(D P_{0} \cdots P_{j} D^{-}\right)^{\prime}\right\}\left(D P_{0} \cdots\right. \\
\left.\cdots P_{i-1} Q_{i}+D P_{0} \cdots P_{i_{*}-1} Q_{i_{*}} \tilde{P}_{i_{*}} \cdots P_{i-1} Q_{i}\right)
\end{gathered}
$$

further, with $M_{i} \cdots M_{i_{*}+1}=: \hat{M}_{i}$,

$$
\begin{aligned}
\tilde{G}_{i+1} \hat{M}_{i}^{-1}= & G_{i+1}\left(I+Q_{i_{*}} \tilde{P}_{i_{*}} \cdots P_{i-1} Q_{i}\right) \\
& -\sum_{j=i_{*}+1}^{i} G_{j} D^{-}\left(D P_{0} \cdots P_{j} D^{-}\right)^{\prime} D P_{0} \cdots Q_{i_{*}} \tilde{P}_{i_{*}} \cdots P_{i-1} Q_{i} \\
& -\sum_{j=i_{*}}^{i} G_{j} D^{-}\left(D P_{0} \cdots Q_{i_{*}} \tilde{P}_{i_{*}} \cdots P_{j} D^{-}\right)^{\prime}\left(D P_{0} \cdots P_{i-1} Q_{i}+D P_{0} \cdots Q_{i_{*}} \tilde{P}_{i_{*}} \cdots P_{i-1} Q_{i}\right) \\
& -\sum_{j=i_{*}+1}^{i} G_{j}\left(\hat{M}_{j}-I\right) \square Q_{i} .
\end{aligned}
$$


The first and last terms are of the form we wanted to have. Compute further

$$
\begin{aligned}
& \tilde{G}_{i+1} \hat{M}_{i}^{-1}=G_{i+1}\left(I+Q_{i_{*}} \tilde{P}_{i_{*}} \cdots P_{i-1} Q_{i}\right)-\sum_{j=i_{*}+1}^{i} G_{j}\left(\hat{M}_{j}-I\right) \square Q_{i} \\
& \quad+\sum_{j=i_{*}+1}^{i} G_{j} D^{-} D P_{0} \cdots P_{j} D^{-}\left(D P_{0} \cdots Q_{i_{*}} \tilde{P}_{i_{*}} D^{-}\right)^{\prime} D P_{0} \cdots P_{i-1} Q_{i} \\
& \quad-\sum_{j=i_{*}}^{i} G_{j} D^{-}\left\{\left(D P_{0} \cdots Q_{i_{*}} \tilde{P}_{i_{*}} D^{-}\right)^{\prime} D P_{0} \cdots P_{j} D^{-}\right. \\
& \left.\quad+D P_{0} \cdots Q_{i_{*}} \tilde{P}_{i_{*}} D^{-}\left(D P_{0} \cdots P_{j} D^{-}\right)^{\prime}\right\}\left(D P_{0} \cdots P_{i-1} Q_{i}+D P_{0} \cdots Q_{i_{*}} \tilde{P}_{i_{*}} \cdots P_{i-1} Q_{i}\right) \\
& =G_{i+1}\left(I+Q_{i_{*}} \tilde{P}_{i_{*}} \cdots P_{i-1} Q_{i}\right)-\sum_{j=i_{*}+1}^{i} G_{j}\left(\hat{M}_{j}-I\right) \square Q_{i} \\
& \quad+\sum_{j=i_{*}+1}^{i}\left(G_{j} P_{0} \cdots P_{j}-G_{j-1}\right) D^{-}\left(D P_{0} \cdots Q_{i_{*}} \tilde{P}_{i_{*}} D^{-}\right)^{\prime} D P_{0} \cdots P_{i-1} Q_{i} \\
& \quad+\sum_{j=i_{*}}^{i} G_{j} P_{0} \cdots Q_{i_{*}} \tilde{P}_{i_{*}} D^{-}\left(D P_{0} \cdots P_{j} D^{-}\right)^{\prime}\left(D P_{0} \cdots P_{i-1} Q_{i}+D P_{0} \cdots Q_{i_{*}} \tilde{P}_{i_{*}} P_{i-1} Q_{i}\right)
\end{aligned}
$$

Finally, we observe that, for $j \geq i_{*}+1$

$$
\begin{aligned}
\left(G_{j} P_{0} \cdots P_{j}-G_{j-1}\right) D^{-} & =G_{i+1} P_{i} P_{i-1} \cdots P_{j}\left(P_{0} \cdots P_{j}-P_{j-1}\right) D^{-} \\
& =G_{i+1} \sum_{l-0}^{i-1} Q_{l} \square D^{-}
\end{aligned}
$$

and, for $j>i_{*}$,

$$
\begin{aligned}
G_{j} P_{0} \cdots P_{i_{*}-1} Q_{i_{*}} & =G_{i+1} P_{i} \cdots P_{j} P_{0} \cdots P_{i_{*}-1} Q_{i_{*}}=G_{i+1} P_{0} \cdots P_{i_{*}-1} Q_{i_{*}} \\
G_{i_{*}} P_{0} \cdots P_{i_{*}-1} Q_{i_{*}} & =G_{i+1} P_{i} \cdots P_{i_{*}} P_{0} \cdots P_{i_{*}-1} Q_{i_{*}}=G_{i+1}\left(P_{0} \cdots P_{i_{*}-1} Q_{i_{*}}-Q_{i_{*}}\right),
\end{aligned}
$$

hence, all terms of $\tilde{G}_{i+1} \hat{M}_{i}^{-1}$ have the wanted form.

\subsection{Proof of Lemma 4.2 by induction}

Let $\tilde{G}_{j}=G_{j} F_{j} \cdots F_{0}$ be true for $j=0,1, \ldots, i$. We have to show the representation

$$
\tilde{G}_{i+1}=G_{i+1} F_{i+1} F_{i} \cdots F_{0}
$$

with a factor of the form

$$
F_{i+1}=I-\sum_{l=0}^{i-1} Q_{l} \mathfrak{A}_{i+1, l} Q_{i},
$$

where $\mathfrak{A}_{i+1, l}$ are certain continuous matrix functions. Compute

$$
\begin{aligned}
& \tilde{G}_{i+1}=\tilde{G}_{i}+\tilde{B}_{i} \tilde{Q}_{i}=G_{i}\left(F_{i} \cdots F_{0}\right)+\tilde{B}_{i}\left(F_{i} \cdots F_{0}\right)^{-1} Q_{i}\left(F_{i} \cdots F_{0}\right), \\
& P_{0} \cdots P_{i-1}\left(F_{i} \cdots F_{0}\right)^{-1} Q_{i}=P_{0} \cdots P_{i-1} F_{0}^{-1} \cdots F_{i}^{-1} Q_{i}=P_{0} \cdots P_{i-1} Q_{i},
\end{aligned}
$$


hence

$$
\begin{aligned}
\tilde{G}_{i+1}( & \left.F_{i} \cdots F_{0}\right)^{-1}=G_{i}+\tilde{B}_{i}\left(F_{i} \cdots F_{0}\right)^{-1} Q_{i} \\
= & G_{i}+\left\{B+A D D^{-} H^{\prime} H^{-1} D\right. \\
& \left.\quad-\sum_{j=1}^{i} G_{j}\left(F_{j} \cdots F_{0}\right) D^{-} H\left(H^{-1} D P_{0} \cdots P_{j} D^{-} H\right)^{\prime} H^{-1} D\right\} P_{0} \cdots P_{i-1} Q_{i} \\
= & G_{i}+B P_{0} \cdots P_{i-1} Q_{i}+G_{0} D^{-} H^{\prime} H^{-1} D P_{0} \cdots P_{i-1} Q_{i} \\
& \quad-\sum_{j=1}^{i} G_{j} F_{j} \cdots F_{0} D^{-} H H^{-1 \prime} D P_{0} \cdots P_{j} P_{0} \cdots P_{i-1} Q_{i} \\
& \quad-\sum_{j=1}^{i} G_{j} F_{j} \cdots F_{0} D^{-}\left(D P_{0} \cdots P_{j} D^{-}\right)^{\prime} D P_{0} \cdots P_{i-1} Q_{i} \\
& \quad-\sum_{j=1}^{i} G_{j} F_{j} \cdots F_{0} D^{-} D P_{0} \cdots P_{j} D^{-} H^{\prime} H^{-1} D P_{0} \cdots P_{i-1} Q_{i} . \\
= & G_{i}+B_{0} P_{0} \cdots P_{i-1} Q_{i}-\sum_{j=1}^{i} G_{j} D^{-}\left(D P_{0} \cdots P_{j} D^{-}\right)^{\prime} D P_{0} \cdots P_{i-1} Q_{i} \\
& \quad-\sum_{j=1}^{i} G_{j}\left(F_{j} \cdots F_{0}-I\right) D^{-}\left(D P_{0} \cdots P_{j} D^{-}\right) D P_{0} \cdots P_{i-1} Q_{i} \\
& -\sum_{j=1}^{i} G_{j} P_{0} \cdots P_{j} D^{-} H^{\prime} H^{-1} D P_{0} \cdots P_{i-1} Q_{i} \\
& +\sum_{j=1}^{i-1} G_{j} F_{j} \cdots F_{0} D^{-} H^{\prime} H^{-1} D P_{0} \cdots P_{i-1} Q_{i} .
\end{aligned}
$$

With $G_{j}\left(F_{j} \cdots F_{0}-I\right)=G_{i+1} P_{i} \cdots P_{j}\left(F_{j} \cdots F_{0}-I\right)=G_{i+1}\left(F_{j} \cdots F_{0}-I\right)$ we derive further that

$$
\begin{aligned}
\tilde{G}_{i+1}( & \left.F_{i} \cdots F_{0}\right)^{-1}=G_{i+1}-\sum_{j=2}^{i} G_{i+1}\left(F_{j} \cdots F_{0}-I\right) D^{-}\left(D P_{0} \cdots P_{j} D^{-}\right)^{\prime} D P_{0} \cdots P_{i-1} Q_{i} \\
& +\sum_{j=2}^{i-1} G_{i+1}\left(F_{j} \cdots F_{0}-I\right) D^{-} H^{\prime} H^{-1} D P_{0} \cdots P_{i-1} Q_{i} \\
& +\sum_{j=1}^{i-1}\left\{G_{j}-G_{j+1} P_{0} \cdots P_{j+1}\right\} D^{-} H^{\prime} H^{-1} D P_{0} \cdots P_{i-1} Q_{i} .
\end{aligned}
$$

Consider the expression

$$
\begin{aligned}
G_{j}- & G_{j+1} P_{0} \cdots P_{j+1}=G_{i+1} P_{i} \cdots P_{j+1}\left\{P_{j}-P_{0} \cdots P_{j+1}\right\} \\
& =G_{i+1} P_{i} \cdots P_{j+1}\left\{P_{j}-P_{0} \cdots P_{j}+P_{0} \cdots P_{j} Q_{j+1}\right\} \\
& =G_{i+1} P_{i} \cdots P_{j+1}\left\{\left(I-P_{0} \cdots P_{j-1}\right) P_{j}+P_{0} \cdots P_{j} Q_{j+1}\right\} .
\end{aligned}
$$

Now it remains to check that

$$
\begin{aligned}
F_{i+1}= & I-\left\{\sum_{j=2}^{i}\left(F_{j} \cdots F_{0}-I\right) D^{-}\left(D P_{0} \cdots P_{j} D^{-}\right)^{\prime}+\sum_{j=2}^{i-1}\left(F_{j} \cdots F_{0}-I\right) D^{-} H^{\prime} H^{-1}\right. \\
& \left.+\sum_{j=1}^{i-1} P_{i} \cdots P_{j+1}\left(\left(I-P_{0} \cdots P_{j-1}\right) P_{j}+P_{0} \cdots P_{j} Q_{j+1}\right) D^{-} H^{\prime} H^{-1}\right\} D P_{0} \cdots P_{i-1} Q_{i}
\end{aligned}
$$


is actually of the form demanded in (6.14). However, this is the case because of

$$
F_{j} \cdots F_{0}-I=-\sum_{l=0}^{j-2} Q_{l} \mathfrak{A}_{j l} Q_{j-1}-\cdots-Q_{0} \mathfrak{A}_{20} Q_{1}
$$

and

$$
\begin{aligned}
P_{i} \cdots & P_{j+1}\left(\left(I-P_{0} \cdots P_{j-1}\right) P_{j}+P_{0} \cdots P_{j} Q_{j+1}\right)= \\
= & P_{i} \cdots P_{j+1}\left(P_{0} \cdots P_{j-2} Q_{j-1}+\cdots+P_{0} Q_{1}+Q_{0}\right) P_{j}+P_{i} \cdots P_{j+1}\left(I-Q_{0}\right) \cdots\left(I-Q_{j}\right) Q_{j+1} \\
= & P_{j} \cdots P_{j+1}\left(\left(I-Q_{0}\right)\left(I-Q_{1}\right) \cdots\left(I-Q_{j-2}\right) Q_{j-1}+\ldots+\left(I-Q_{0}\right) Q_{1}+Q_{0}\right) P_{j} \\
& \quad+P_{i} \cdots P_{j+1}\left(Q_{j+1}-\left(Q_{0}+Q_{1}+\ldots Q_{j}\right) Q_{j+1}+\ldots\right) \\
= & \left(\left(I-Q_{0}\right) \cdots\left(I-Q_{j-2}\right) Q_{j-1}+\ldots+\left(I-Q_{0}\right) Q_{1}+Q_{0}\right) P_{j} \\
& -\left(Q_{0}+Q_{1}+\ldots+Q_{j}\right) Q_{j+1}+\ldots
\end{aligned}
$$

that is, from the last expression for $j=i-1$ there arises a term in $F_{i+1}$ with $-Q_{i-1} Q_{i}$ on the top left, while the other terms in $F_{i+1}$ begin with $Q_{j}, j \leq i-2$. Consequently, $F_{i+1}$ is of the form

$$
F_{i+1}=I-Q_{i-1} \mathfrak{A}_{i+1, i-1} Q_{i}-\ldots Q_{0} \mathfrak{A}_{i+1,0} Q_{i}
$$

with certain matrices $\mathfrak{A}_{i+1, l}$ as requested.

\section{References}

[BaMä] K. Balla und R. März: A unified approach to linear differential algebraic equations and their adjoint equations. Humboldt-Universität Berlin, Institut für Mathematik, Preprint 2000-18.

[Bo] Yu. Boyarintsev: Methods of solving singular systems of ordinary differential equations. John Wiley \& Sons, 1992 (Russian original: 1988, Nauka, Siberian Division).

[BrCaPe] K.E. Brenan, S.L. Campbell, L.R. Petzold: Numerical solution of initialvalue problems in differential-algebraic equations. Elsevier Science Publ. Co, Inc. 1989.

[Ca] S.L. Campbell: A general form for solvable linear time varying singular systems of differential equations. SIAM J. Math. Anal. 18(4) 1987, 1101-1115.

[CaPe] S.L. Campbell, R.L. Petzold: Canonical forms and solvable singular systems of differential equations. SIAM J. Alg. Discr. Methods 4, 1983, 517-521.

[CoCa] E. A. Coddington, R. Carlson: Linear ordinary differential equations. SIAM Philadelphia 1997.

[Es et all] D. Estévez Schwarz, U. Feldmann, R. März, S. Sturtzel, C. Tischendorf: Finding beneficial DAE structures in circuit simulation. To appear in a special volume at Springer concerning mathematics for solving problems in industry and economy. 
[Gaj] I.V. Gajshun: Vvedenie v teoriyu linejnykh nestadzhionarnykh sistem. NAN Belarusi, Minsk 1999.

[Ga] F.R. Gantmacher: Teoriya matrits. Moskva, Nauka 1966.

[GePe] C.W. Gear and L.R. Petzold: ODE methods for the solution of differentialalgebraic systems. SIAM J. Numer. Anal. 21(4) 1984, 716-728.

[GrMä] E. Griepentrog, R. März: Basic properties of some differential-algebraic equations. Zeitschrift für Analysis und ihre Anwendungen 8, 1989, 25-40.

[GrMä1] E. Griepentrog, R. März: Differential-algebraic equations and their numerical tratment. Teubner, Leipzig, 1986.

[Ha] B. Hansen: Linear time-varying differential-algebraic equations being tractable with the index $k$. Humboldt-Universität Berlin, Institut für Mathematik, Preprint 246, 1990.

[HiMäTi] I. Higueras and R. März, C. Tischendorf: Numerically well formulated index-1DAEs. Humboldt-Universität Berlin, Institut für Mathematik, Preprint $2001-5$.

[KuMe] R. Kunkel, V. Mehrmann: Canonical forms for linear differential-algebraic equations with variable coefficients. J. Comput. Appl. Math. 56, 1994, 225-251.

[Mä] R. März: Differential algebraic systems anew. To appear in Applied Numerical Mathematics.

[Mä1] R. März: Some results concerning index-3 differential-algebraic equations. J. Mathem. Analysis and Applications 140(1) 1989, 177-199.

[Mä2] R. März: Numerical methods for differential-algebraic equations. Acta Numerica 1992, 141-198.

[Mä3] R. März: Adjoint equations of differential-algebraic systems and optimal control problems. Proc. of the Institute of Mathematics, NAS of Belarus, Minsk, Vol. 7 2001.

[ReMaBa] G. Reißig, W.S. Martinson, P.J. Barton: Differential-algebraic equations of index 1 may have an arbitrarily high structural index. SIAM J. Scie. Comp. 21(6)2000, 1987-1990.

[RiMä] R. Riaza, R. März: Singularities of linear time-varying DAEs. HumboldtUniversität Berlin, Institut für Mathematik, Preprint 2001-9, 2001.

[RaRh] P.J. Rabier, W.C. Rheinboldt: Classical and generalized solutions of time-dependent linear differential-algebraic equations. Linear Algebra and its Applications 2145, 1996, 259-293.

[Schu] I. Schumilina: Index-3 DAEs with properly stated leading term. In preparation. 